\title{
Anisotropic mean curvature flow for two-dimensional surfaces in higher codimension: a numerical scheme
}

\author{
PAOLA POZZI ${ }^{\dagger}$ \\ Abteilung für Angewandte Mathematik, Albert-Ludwigs-Universität Freiburg i. Br., \\ Hermann-Herder Strasse 10, D-79104 Freiburg i. Br., Germany
}

[Received 17 July 2007 and in revised form 3 March 2008]

We consider evolution of two-dimensional parametric surfaces by anisotropic mean curvature in $\mathbb{R}^{n}$, for arbitrary $n \geqslant 3$. After deriving a classical and a weak formulation of the flow, a fully discrete stable finite element scheme is proposed, and numerical tests and simulations are presented.

\section{Introduction}

In this paper we consider evolution of parametric two-dimensional surfaces by anisotropic mean curvature in arbitrary codimension. After deriving a classical and a weak formulation of the flow, we discretize the problem by the finite element method, provide a fully discrete stable scheme and present numerical tests and examples.

Anisotropic mean curvature flow has been widely studied, both analytically and numerically. Much of the research concentrated so far on the case of codimension one, i.e. on the flow for hypersurfaces. Given the amount of work done in this field, we do not attempt to give a list of references but limit ourselves to point to the survey article [9]. There the interested reader can get a flavour of recent progress on some important topics concerning the theoretical and numerical analysis of the mean curvature flow, and the list of references helps finding many of the most important contributions in the field; applications are also briefly discussed.

For the case of higher codimension there are some works in the isotropic setting (see for example [22] and [2]), but the anisotropic case seems to have been less studied. The evolution by anisotropic mean curvature of parametric curves in $\mathbb{R}^{n}$ is investigated by the author in [15]; in [18] a level set approach to motion of manifolds of arbitrary codimension is studied. The survey article [14], on the existence and regularity theory for Cartan functionals (i.e. for general parameter invariant double integrals defined on parametric surfaces with arbitrary codimension), can also be considered as related to our studies.

The aim of this work is to extend the algorithm given in [7, §4.2] for the anisotropic mean curvature flow of parametric surfaces in $\mathbb{R}^{3}$ to the the case of arbitrary codimension. The generalization is not quite straightforward, since the formulation of the three-dimensional flow already heavily relies on the fact that the codimension is equal to one.

An extension of the algorithm to arbitrary codimension is made possible by embedding the problem in the field of Minkowski (and its related Finsler) geometry. In this context the Euclidean space $\mathbb{R}^{n}$ is endowed with an additional norm which defines the anisotropic geometry of the space. Concepts of area and content are well defined and come with a range of powerful tools (see for example the beautiful book by Thompson [20], and [1]).

\footnotetext{
†E-mail: paola@mathematik.uni-freiburg.de
} 
Similarly to [3] we describe the anisotropic area by introducing an anisotropy function $F$ defined on $\Lambda^{2} \mathbb{R}^{n}$ : loosely speaking, $F$ provides a weight for each tangent space to the surface $M$. A natural correspondence between a tangent space and its unit normal in the three-dimensional case allows us to recover the more familiar definitions used for the flow in codimension one (see for example [9, §8] and [6]). In the context of Minkowski geometry, $F$ can be interpreted as a "choice of area functional" in $\mathbb{R}^{n}$ seen as a normed space (see [15, §1], [20], [1]).

A proper definition of the energy functional that we will consider in this work is given in Section 2. In Section 3 we briefly review a few concepts connected with exterior powers and simple $k$-vectors in order to help the reader gain some geometrical intuition. A number of interesting problems come up if the anisotropy function $F$ is defined only on the set of simple vectors $\Lambda_{s}^{2} \mathbb{R}^{n}$ instead of the whole of $\Lambda^{2} \mathbb{R}^{n}$; a few references are given for those readers who would like to "plunge" deeper in this direction.

After calculating the first variation of the energy functional in Section 4 we give a definition of the anisotropic mean curvature vector $H_{F}$ (see Definition 4.1) and anisotropic mean curvature flow (see Definition 4.3). The latter has the form

$$
\frac{\partial \mathbf{x}}{\partial t}=H_{F}
$$

where $\mathbf{x}(t, \cdot)$ is an immersion of a fixed two-dimensional compact manifold $\tilde{M}$ into $\mathbb{R}^{n}$. Aiming at deriving a numerical scheme, we give in Section 5 a weak formulation of the flow. A discretization by the finite element method follows in Section 6. Here two schemes are presented: the first one (Algorithm 6.1) is a generalization to higher codimension of the algorithm given in [7, §4.2], and the second one (Algorithm 6.3 is a modification of the first one in order to guarantee stability. A proof of the stability of Algorithm 6.3 is presented in Section 7 In Section 8 we consider numerical tests that confirm our claim on the necessity of introducing a stabilizing term, and we provide graphical examples. Finally, in Section 9, we generalize the definition of Wulff shape to the case of higher codimension (in $\mathbb{R}^{3}$ Wulff shapes play an important role since they are solutions to an isoperimetric problem) and recognize an ongoing interplay between dual structures; the latter is a typical phenomenon in Minkowski spaces.

\section{Anisotropic area functional}

DEFinition 2.1 Consider an oriented two-dimensional closed compact surface $M$ in $\mathbb{R}^{n}, n \geqslant 3$, and choose a partition of unity $\left\{\rho_{\beta}\right\}_{\beta \in \mathcal{I}}$ with each $\rho_{\beta}$ supported in an open set $U_{\beta} \subset M$ which is the image of an oriented coordinate chart $\varphi_{\beta}$ from an open set $W_{\beta} \subset \mathbb{R}^{2}$. For each $\beta$ there are coordinate tangent vectors $\partial_{i}^{\beta}=D \varphi_{\beta}\left(e_{i}\right)$ for $i=1,2$. Let $F: \Lambda^{2} \mathbb{R}^{n} \rightarrow \mathbb{R}$ be an anisotropy function as in Definition 2.2 below. The map $F$ defines an anisotropic area functional given by

$$
|M|_{F}=\sum_{\beta \in \mathcal{I}} \int_{y \in W_{\beta}} \rho_{\beta} \circ \varphi_{\beta} F\left(\partial_{1}^{\beta} \wedge \partial_{2}^{\beta}\right) \mathrm{d} y_{1} \mathrm{~d} y_{2} .
$$

As pointed out in [3], the homogeneity of $F$ guarantees that this is independent of the choice of the oriented charts and partition of unity. The anisotropic area element is defined by $d \mu_{F}=$ $F\left(\partial_{1}^{\beta} \wedge \partial_{2}^{\beta}\right) \mathrm{d} y_{1} d y_{2}$. 
DEFINITION 2.2 By an anisotropy function $F$ we understand a positive convex function $F$ : $\Lambda^{2} \mathbb{R}^{n} \rightarrow[0, \infty)$ such that $F \in C^{2}\left(\Lambda^{2} \mathbb{R}^{n} \backslash\{0\}\right), F\left(p_{1} \wedge p_{2}\right)>0$ for $p_{1} \wedge p_{2} \neq 0$ and $F$ is positively homogeneous of degree one,

$$
F\left(\lambda p_{1} \wedge p_{2}\right)=\lambda F\left(p_{1} \wedge p_{2}\right) \quad \text { for } \lambda>0 .
$$

$F$ is said to be reversible if $F\left(-p_{1} \wedge p_{2}\right)=F\left(p_{1} \wedge p_{2}\right)$ for all $p_{1} \wedge p_{2} \in \Lambda^{2} \mathbb{R}^{n}$.

Note that a reversible anisotropy function is nothing else than a smooth norm on the $n(n-1) / 2$ dimensional vector space $\Lambda^{2} \mathbb{R}^{n}$.

It is not difficult to verify that (2.2) implies

$$
\begin{array}{ll}
\left.D F\right|_{p_{1} \wedge p_{2}}\left(p_{1} \wedge p_{2}\right)=F\left(p_{1} \wedge p_{2}\right), & \left.D^{2} F\right|_{p_{1} \wedge p_{2}}\left(p_{1} \wedge p_{2}\right)\left(q_{1} \wedge q_{2}\right)=0, \\
\left.D F\right|_{\lambda p_{1} \wedge p_{2}}=\left.D F\right|_{p_{1} \wedge p_{2}}, & \left.D^{2} F\right|_{\lambda p_{1} \wedge p_{2}}=\left.\frac{1}{\lambda} D^{2} F\right|_{p_{1} \wedge p_{2}},
\end{array}
$$

for all $p_{1} \wedge p_{2} \in \Lambda^{2} \mathbb{R}^{n} \backslash\{0\}, q_{1} \wedge q_{2} \in \Lambda^{2} \mathbb{R}^{n}$ and $\lambda>0$.

Typical choices for $F$ can be found in Section 8 , for the special case of $n=3$ further examples of anisotropy functions are given in [9, §8.1].

\section{Preliminaries and notation}

Recall the following general facts. For $X$ an $n$-dimensional vector space over $\mathbb{R}$, we denote by $\Lambda^{k} X$ its exterior power and by $\Lambda_{s}^{k} X$ the set of all simple $k$-vectors $v_{1} \wedge \cdots \wedge v_{k}$, where $v_{1}, \ldots, v_{k}$ are vectors in $X$ (see [21] for basic definitions of exterior algebra and related topics). The following important properties will help the reader give a geometrical meaning to purely algebraic objects such as $k$-vectors.

THEOREM 3.1 (i) A system of vectors $\left\{v_{1}, \ldots, v_{p}\right\}$ of $X$ is linearly dependent if and only if $v_{1} \wedge \cdots \wedge v_{p}=0$.

(ii) Assume that in each of the systems $\left\{v_{1}, \ldots, v_{p}\right\}$ and $\left\{w_{1}, \ldots, w_{p}\right\}$ the vectors are linearly independent. Then $\operatorname{span}\left(v_{1}, \ldots, v_{p}\right)=\operatorname{span}\left(w_{1}, \ldots, w_{p}\right)$ if and only if the $p$-vectors $v_{1} \wedge$ $\cdots \wedge v_{p}$ and $w_{1} \wedge \cdots \wedge w_{p}$ are proportional.

Proof. A proof can be found in [21, Theorem 8.44].

If we endow $X$ with a Euclidean structure determined by an inner product then $\Lambda^{k} X$ can also be given an inner product $\langle\cdot, \cdot\rangle$ defined for simple elements $a=v_{1} \wedge \cdots \wedge v_{k}$ and $b=w_{1} \wedge \cdots \wedge w_{k}$ by

$$
\langle a, b\rangle=\operatorname{det}\left(v_{i} \cdot w_{j}\right)
$$

and extended bilinearly to the whole of $\Lambda^{k} X$. Naturally the Euclidean norm is defined by $|a|=$ $\sqrt{\langle a, a\rangle}$. We furthermore remark that if $\left\{e_{1}, \ldots, e_{n}\right\}$ is an orthonormal basis for $X$, then the $k$-vectors

$$
e_{i_{1}} \wedge \cdots \wedge e_{i_{k}}, \quad 1 \leqslant i_{1}<\cdots<i_{k} \leqslant n,
$$

form an orthonormal basis of $\Lambda^{k} X$.

Note that $\Lambda^{k} X$ is by definition a vector space of dimension $\operatorname{dim} \Lambda^{k} X=\left(\begin{array}{l}n \\ k\end{array}\right)$, whereas $\Lambda_{s}^{k} X$ is just an algebraic cone, often referred to as the Grassmann cone. The projectivization of this cone 
is called the Grassmann variety and is denoted by $\mathrm{Gr}_{k}(X)$ (see [21, p. 318]). By Theorem 3.1, the points of $\mathrm{Gr}_{k}(X)$ are in one-to-one correspondence with the $k$-dimensional subspaces of $X$.

As observed in [1], to define a $k$-dimensional anisotropic area functional we do not need to define the "norm" $F$ on all of $\Lambda^{k} X$ but it suffices to define it on $\Lambda_{s}^{k} X$. Observe that for $k=1, n-1$ every $k$-vector is simple and therefore $\Lambda_{s}^{k} X$ preserves the nice structure of a vector space and makes the analysis much more approachable. In fact these special cases have been much investigated, the first $(k=1)$ relating to the anisotropic energy functional employed to define the anisotropic curve shortening flow studied in [15], and the second $(k=n-1)$ corresponding to the anisotropic energy used to describe the anisotropic mean curvature flow for hypersurfaces (see [9], [6] and [3]).

Here we are interested in the case where $k=2$, for which $\Lambda_{s}^{k} X$ is only a set. A discussion of various complications induced by having $F$ defined only on a set of simple vectors can be found in [1]. There it is pointed out that one of the biggest issues is that "it is not clear how to write the triangle inequality, and, even if an apparent analogue could be found, it would have to be justified in terms of its geometric significance" ([1, p. 18]). Further helpful comments on the topic can be found in [20, Chapters 5 and 6]. A reader interested in this issue will find that a number of interesting geometric problems and open questions await further investigation.

In the following we consider the case where $X=\mathbb{R}^{n}$ (endowed with the standard Euclidean inner product and orthonormal oriented basis $\left.\left\{e_{1}, \ldots, e_{n}\right\}\right)$ and $k=2$. To simplify the analysis and avoid the tricky question of what is meant by convexity when $F$ is defined only on the cone of simple vectors, we assume that $F$ is convex on the whole exterior power $\Lambda^{2} \mathbb{R}^{n}$ (recall Definition 2.2).

The isotropic case is recovered by choosing $F\left(p_{1} \wedge p_{2}\right)=\left|p_{1} \wedge p_{2}\right|$, i.e. when $F$ measures the area of the parallelogram spanned by the vectors $p_{1}$ and $p_{2}$.

Finally, let us remark that if $n=3$ we can use the Hodge operator $*$ (induced by the canonical inner product on $\mathbb{R}^{3}$ and the oriented basis $\left.\left\{e_{1}, e_{2}, e_{3}\right\}\right)$ to single out a unique isomorphism between the three-dimensional vector spaces $\Lambda^{2} \mathbb{R}^{3}$ and $\Lambda^{1} \mathbb{R}^{3}=\mathbb{R}^{3}$ and define a map $\tilde{F}: \mathbb{R}^{3} \rightarrow \mathbb{R}$ by $\tilde{F}=F \circ *$. (More on the Hodge operator can be found in [23, §3.8] and [12, §2.7].) This allows us to recover a more familiar formulation of the anisotropic area functional, namely

$$
|M|_{F}=\sum_{\beta \in \mathcal{I}} \int_{y \in W_{\beta}} \rho_{\beta} \circ \varphi_{\beta} F\left(\frac{\partial_{1}^{\beta} \wedge \partial_{2}^{\beta}}{\left|\partial_{1}^{\beta} \wedge \partial_{2}^{\beta}\right|}\right)\left|\partial_{1}^{\beta} \wedge \partial_{2}^{\beta}\right| \mathrm{d} y_{1} \mathrm{~d} y_{2}=\int_{M} \tilde{F}(v) \mathrm{d} \mathcal{H}^{2}
$$

where $v=* \frac{\partial_{1}^{\beta} \wedge \partial_{2}^{\beta}}{\left|\partial_{1}^{\beta} \wedge \partial_{2}^{\beta}\right|}$ is the unit normal to $M$ with direction determined by the orientation, and $\mathcal{H}^{2}$ is the two-dimensional Hausdorff measure.

Notation. We use $v \cdot w$ to denote the inner product in $\mathbb{R}^{n}$, and $\langle\cdot, \cdot\rangle$ to denote the induced inner product in $\Lambda^{2} \mathbb{R}^{n}$.

We write $\nabla F, \mathcal{H}_{F}$ for the gradient vector and Hessian matrix of the map $F$ with respect to the canonical orthonormal basis in $\Lambda^{2} \mathbb{R}^{n}$ (induced by the canonical basis and inner product in $\mathbb{R}^{n}$ ). Thus we have

$$
\begin{aligned}
& \left.D F\right|_{p_{1} \wedge p_{2}}\left(w_{1} \wedge w_{2}\right)=\left\langle\nabla F\left(p_{1} \wedge p_{2}\right), w_{1} \wedge w_{2}\right\rangle \\
& \left.D^{2} F\right|_{p_{1} \wedge p_{2}}\left(w_{1} \wedge w_{2}\right)\left(q_{1} \wedge q_{2}\right)=\left\langle\mathcal{H}_{F}\left(p_{1} \wedge p_{2}\right) w_{1} \wedge w_{2}, q_{1} \wedge q_{2}\right\rangle .
\end{aligned}
$$




\section{First variation and definition of the AMCF}

First of all let us make a couple of remarks which will be useful throughout the subsequent calculations. Due to the compactness of the surface $M$ we can assume that the index set $\mathcal{I}$ of the open covering $\left\{U_{\beta}\right\}_{\beta \in \mathcal{I}}$ for $M$ is finite and that the functions $\rho_{\beta}$ of the partition of unity have the additional property of being compactly supported in $U_{\beta}$ (see for example [16, Theorem 29.3]).

Recall also that for any two coordinate charts $\varphi_{\alpha}: W_{\alpha} \rightarrow U_{\alpha}, \varphi_{\beta}: W_{\beta} \rightarrow U_{\beta}$, whose images have non-empty intersection $U_{\alpha} \cap U_{\beta}$, one can easily show that $\partial_{1}^{\alpha} \wedge \partial_{2}^{\alpha}=\operatorname{det}\left(\varphi_{\beta}^{-1} \circ \varphi_{\alpha}\right) \partial_{1}^{\beta} \wedge \partial_{2}^{\beta}$. Thus two coordinate charts share the same orientation if the 2-vectors $\partial_{1}^{\alpha} \wedge \partial_{2}^{\alpha}$ and $\partial_{1}^{\beta} \wedge \partial_{2}^{\beta}$ are positive multiples of each other.

Next we calculate the first variation for $|M|_{F}$.

THEOREM 4.1 Let $M$ be as in Definition 2.1. and take $\mathcal{O}$ to be an open subset of $\mathbb{R}^{n}$ such that $\mathcal{O} \cap M \neq \emptyset$ and define a one-parameter family of diffeomorphisms $\phi_{t}: \mathcal{O} \rightarrow \mathcal{O}$ such that

$$
\left\{\begin{array}{l}
\text { (A) } \phi(t, x)=\phi_{t}(x) \text { is a smooth map: }(-1,1) \times \mathcal{O} \rightarrow \mathcal{O}, \\
\text { (B) } \phi_{0}(x)=x \text { for } x \in \mathcal{O}, \\
\text { (C) } \phi_{t}(x)=x \text { for all } t \in(-1,1), x \in \mathcal{O} \backslash K,
\end{array}\right.
$$

where $K$ is a compact subset of $\mathcal{O}$. We denote the initial velocity vector by

$$
\xi(x)=\left.\frac{\partial \phi(t, x)}{\partial t}\right|_{t=0} .
$$

Let $M^{t}=\phi_{t}(M)$ (where we set $\phi_{t}(x)=x$ for $x \in M \backslash(M \cap K)$ ). Then

$$
\left.\frac{\mathrm{d}}{\mathrm{d} t}\right|_{t=0}\left|M^{t}\right|_{F}=-\int_{M} \xi \cdot H_{F} \mathrm{~d} A,
$$

where $H_{F}$ is the anisotropic mean curvature vector and $\mathrm{d} A$ is the area element on $M$.

DEFINITION 4.1 The anisotropic mean curvature vector $H_{F}$ is a vector normal to the surface $M$. In local coordinates it is described by

$$
H_{F}:=\sum_{i=1}^{n-2} \frac{1}{\left|\partial_{1} \wedge \partial_{2}\right|}\left(\partial_{1}\left(\left.D F\right|_{\partial_{1} \wedge \partial_{2}}\right)\left(v_{i} \wedge \partial_{2}\right)+\partial_{2}\left(\left.D F\right|_{\partial_{1} \wedge \partial_{2}}\right)\left(\partial_{1} \wedge v_{i}\right)\right) v_{i},
$$

where $\left\{v_{1}, \ldots, v_{n-2}\right\}$ is any orthonormal frame normal to $M$.

In Lemma 4.3 below we show that the above definition is correct.

Proof of Theorem 4.1. By definition $M^{t}$ is a one-parameter family of manifolds such that $M^{0}=M$ and $M^{t}$ agrees with $M$ outside some compact subset of $\mathcal{O}$. The map $\xi$ is obviously supported in a compact subset of $\mathcal{O}$.

Note that if $\left\{\varphi_{\beta}\right\}_{\beta \in \mathcal{I}}, \varphi_{\beta}: W_{\beta} \rightarrow U_{\beta} \subset M$, is an oriented atlas for $M$, then $\left\{\tilde{\varphi}_{\beta}^{t}\right\}_{\beta \in \mathcal{I}}$ with

$$
\tilde{\varphi}_{\beta}^{t}:=\left.\phi_{t}\right|_{M} \circ \varphi_{\beta}: W_{\beta} \rightarrow U_{\beta}^{t}:=\phi_{t} \circ \varphi_{\beta}\left(W_{\beta}\right)
$$

is an oriented atlas for $M^{t}$. Moreover, $\left\{\tilde{\rho}_{\beta}^{t}\right\}_{\beta \in \mathcal{I}}$ with

$$
\tilde{\rho}_{\beta}^{t}:=\rho_{\beta} \circ\left(\left.\phi_{t}\right|_{M}\right)^{-1}
$$


is a partition of unity subordinate to $U_{\beta}^{t}$. Therefore

$$
\begin{aligned}
\left|M^{t}\right|_{F} & =\sum_{\beta \in \mathcal{I}} \int_{y \in W_{\beta}} \tilde{\rho}_{\beta}^{t} \circ \tilde{\varphi}_{\beta}^{t} F\left(\partial_{1}^{\beta, t} \wedge \partial_{2}^{\beta, t}\right) \mathrm{d} y_{1} \mathrm{~d} y_{2} \\
& =\sum_{\beta \in \mathcal{I}} \int_{y \in W_{\beta}} \rho_{\beta} \circ \varphi_{\beta} F\left(\partial_{1}^{\beta, t} \wedge \partial_{2}^{\beta, t}\right) \mathrm{d} y_{1} \mathrm{~d} y_{2},
\end{aligned}
$$

where $\partial_{i}^{\beta, t}=D \tilde{\varphi}_{\beta}^{t}\left(e_{i}\right)=D\left(\phi_{t} \circ \varphi_{\beta}\right)\left(e_{i}\right)$ for $i=1,2$. More precisely, at $x=\phi_{t} \circ \varphi_{\beta}(y) \in M^{t}$ we have $\partial_{i}^{\beta, t}(x)=\frac{\partial}{\partial y_{i}}\left(\phi_{t} \circ \varphi_{\beta}\right)(y)$.

To compute $\left.\frac{\mathrm{d}}{\mathrm{d} t}\right|_{t=0}\left|M^{t}\right|_{F}$ we need to calculate

$$
\left.\frac{\mathrm{d}}{\mathrm{d} t}\right|_{t=0} F\left(\partial_{1}^{\beta, t} \wedge \partial_{2}^{\beta, t}\right) .
$$

Since $\left.\frac{\mathrm{d}}{\mathrm{d} t}\right|_{t=0} \partial_{i}^{\beta, t}=\frac{\partial}{\partial y_{i}}\left(\xi \circ \varphi_{\beta}\right)(y)=\partial_{i}^{\beta} \xi$ we can write

$$
\begin{aligned}
& \left.\frac{\mathrm{d}}{\mathrm{d} t}\right|_{t=0} F\left(\partial_{1}^{\beta, t} \wedge \partial_{2}^{\beta, t}\right)=\left.D F\right|_{\partial_{1}^{\beta} \wedge \partial_{2}^{\beta}}\left(\partial_{1}^{\beta} \xi \wedge \partial_{2}^{\beta}+\partial_{1}^{\beta} \wedge \partial_{2}^{\beta} \xi\right) \\
& =\left.D F\right|_{\partial_{1}^{\beta} \wedge \partial_{2}^{\beta}}\left(\partial_{1}^{\beta}\left(\xi \wedge \partial_{2}^{\beta}\right)-\xi \wedge \partial_{1}^{\beta} \partial_{2}^{\beta}+\partial_{2}^{\beta}\left(\partial_{1}^{\beta} \wedge \xi\right)-\partial_{2}^{\beta} \partial_{1}^{\beta} \wedge \xi\right) \\
& =\left.D F\right|_{\partial_{1}^{\beta} \wedge \partial_{2}^{\beta}}\left(\partial_{1}^{\beta}\left(\xi \wedge \partial_{2}^{\beta}\right)+\partial_{2}^{\beta}\left(\partial_{1}^{\beta} \wedge \xi\right)\right) \\
& =\partial_{1}^{\beta}\left(\left.D F\right|_{\partial_{1}^{\beta} \wedge \partial_{2}^{\beta}}\left(\xi \wedge \partial_{2}^{\beta}\right)\right)+\partial_{2}^{\beta}\left(\left.D F\right|_{\partial_{1}^{\beta} \wedge \partial_{2}^{\beta}}\left(\partial_{1}^{\beta} \wedge \xi\right)\right) \\
& -\partial_{1}^{\beta}\left(\left.D F\right|_{\partial_{1}^{\beta} \wedge \partial_{2}^{\beta}}\right)\left(\xi \wedge \partial_{2}^{\beta}\right)-\partial_{2}^{\beta}\left(\left.D F\right|_{\partial_{1}^{\beta} \wedge \partial_{2}^{\beta}}\right)\left(\partial_{1}^{\beta} \wedge \xi\right) \text {. }
\end{aligned}
$$

Therefore

$$
\begin{aligned}
& \left.\frac{\mathrm{d}}{\mathrm{d} t}\right|_{t=0}\left|M^{t}\right|_{F}=\left.\sum_{\beta \in \mathcal{I}} \int_{y \in W_{\beta}} \rho_{\beta} \circ \varphi_{\beta} \frac{\mathrm{d}}{\mathrm{d} t}\right|_{t=0} F\left(\partial_{1}^{\beta, t} \wedge \partial_{2}^{\beta, t}\right) \mathrm{d} y_{1} \mathrm{~d} y_{2} \\
& =\sum_{\beta \in \mathcal{I}} \int_{y \in W_{\beta}} \rho_{\beta} \circ \varphi_{\beta}\left\{\frac{\partial}{\partial y_{1}}\left(\left.D F\right|_{\partial_{1}^{\beta} \wedge \partial_{2}^{\beta}}\left(\left(\xi \circ \varphi_{\beta}\right)(y) \wedge \frac{\partial \varphi_{\beta}}{\partial y_{2}}(y)\right)\right)\right. \\
& \left.+\frac{\partial}{\partial y_{2}}\left(\left.D F\right|_{\partial_{1}^{\beta} \wedge \partial_{2}^{\beta}}\left(\frac{\partial \varphi_{\beta}}{\partial y_{1}}(y) \wedge\left(\xi \circ \varphi_{\beta}\right)(y)\right)\right)\right\} \mathrm{d} y_{1} \mathrm{~d} y_{2} \\
& -\sum_{\beta \in \mathcal{I}} \int_{y \in W_{\beta}} \rho_{\beta} \circ \varphi_{\beta}\left\{\frac{\partial}{\partial y_{1}}\left(\left.D F\right|_{\partial_{1}^{\beta} \wedge \partial_{2}^{\beta}}\right)\left(\left(\xi \circ \varphi_{\beta}\right)(y) \wedge \frac{\partial \varphi_{\beta}}{\partial y_{2}}(y)\right)\right. \\
& \left.+\frac{\partial}{\partial y_{2}}\left(\left.D F\right|_{\partial_{1}^{\beta} \wedge \partial_{2}^{\beta}}\right)\left(\frac{\partial \varphi_{\beta}}{\partial y_{1}}(y) \wedge\left(\xi \circ \varphi_{\beta}\right)(y)\right)\right\} \mathrm{d} y_{1} \mathrm{~d} y_{2}=I+I I .
\end{aligned}
$$

The velocity vector $\xi$ can be written as sum of its tangent and normal components, $\xi=\xi^{T}+\xi^{N}$, where $\xi^{T}=\xi_{1}^{T} \partial_{1}+\xi_{2}^{T} \partial_{2}$ and $\xi^{N}=\sum_{i=1}^{n-2}\left(\xi \cdot v_{i}\right) v_{i}$ for $\left\{v_{1}, \ldots, v_{n-2}\right\}$ an orthonormal frame in $(T . M)^{\perp}$. 
We first consider the integral $I$. Omitting the indices $\beta$ for simplicity, the integrand can be written as

$$
\begin{aligned}
\rho\left\{\partial_{1}\left(\left.D F\right|_{\partial_{1} \wedge \partial_{2}}\left(\xi \wedge \partial_{2}\right)\right)+\right. & \left.\partial_{2}\left(\left.D F\right|_{\partial_{1} \wedge \partial_{2}}\left(\partial_{1} \wedge \xi\right)\right)\right\} \\
= & \rho\left\{\partial_{1}\left(\left.D F\right|_{\partial_{1} \wedge \partial_{2}}\left(\xi^{T} \wedge \partial_{2}\right)\right)+\partial_{2}\left(\left.D F\right|_{\partial_{1} \wedge \partial_{2}}\left(\partial_{1} \wedge \xi^{T}\right)\right)\right\} \\
& +\rho\left\{\partial_{1}\left(\left.D F\right|_{\partial_{1} \wedge \partial_{2}}\left(\xi^{N} \wedge \partial_{2}\right)\right)+\partial_{2}\left(\left.D F\right|_{\partial_{1} \wedge \partial_{2}}\left(\partial_{1} \wedge \xi^{N}\right)\right)\right\}=W_{1}+W_{2} .
\end{aligned}
$$

Using (2.3), the fact that $\partial_{i} \wedge \partial_{i}=0$, the linearity of $\left.D F\right|_{p_{1} \wedge p_{2}} \in L\left(\Lambda^{2} \mathbb{R}^{n}, \mathbb{R}\right)$, and denoting by $\sqrt{g}$ the area element

$$
\sqrt{g}=\sqrt{\operatorname{det}\left(\left(g_{i j}\right)_{i, j=1,2}\right)}=\sqrt{\operatorname{det}\left(\left(\partial_{i} \cdot \partial_{j}\right)_{i, j=1,2}\right)}=\left|\partial_{1} \wedge \partial_{2}\right|
$$

we obtain for the first term

$$
\begin{aligned}
W_{1} & =\rho\left\{\partial_{1}\left(\left.D F\right|_{\partial_{1} \wedge \partial_{2}}\left(\xi_{1}^{T} \partial_{1} \wedge \partial_{2}\right)\right)+\partial_{2}\left(\left.D F\right|_{\partial_{1} \wedge \partial_{2}}\left(\partial_{1} \wedge \xi_{2}^{T} \partial_{2}\right)\right)\right\} \\
& =\rho\left\{\partial_{1}\left(\xi_{1}^{T} F\left(\partial_{1} \wedge \partial_{2}\right)\right)+\partial_{2}\left(\xi_{2}^{T} F\left(\partial_{1} \wedge \partial_{2}\right)\right)\right\} \\
& =\rho \sqrt{g}\left\{\frac{1}{\sqrt{g}} \partial_{1}\left(\sqrt{g} \xi_{1}^{T} F\left(\frac{\partial_{1} \wedge \partial_{2}}{\left|\partial_{1} \wedge \partial_{2}\right|}\right)\right)+\frac{1}{\sqrt{g}} \partial_{2}\left(\sqrt{g} \xi_{2}^{T} F\left(\frac{\partial_{1} \wedge \partial_{2}}{\left|\partial_{1} \wedge \partial_{2}\right|}\right)\right)\right\} \\
& =\rho \sqrt{g} \operatorname{div}_{M}\left(F\left(\frac{\partial_{1} \wedge \partial_{2}}{\left|\partial_{1} \wedge \partial_{2}\right|}\right) \xi^{T}\right) .
\end{aligned}
$$

(Recall the formula for the divergence of a tangential vector field on a manifold, see for example [19, Chapter 2, (2.12)].) Note that by Theorem 3.1 any two equioriented bases $\left\{\partial_{1}, \partial_{2}\right\}$ and $\left\{\tilde{\partial}_{1}, \tilde{\partial}_{2}\right\}$ of the same tangent space have equal ratio $\frac{\partial_{1} \wedge \partial_{2}}{\left|\partial_{1} \wedge \partial_{2}\right|}=\frac{\tilde{\partial}_{1} \wedge \tilde{\partial}_{2}}{\left|\tilde{\partial}_{1} \wedge \tilde{\partial}_{2}\right|}$. Thus the above expression is independent of the coordinates chosen.

For the second term $W_{2}$ we observe that

$$
\begin{aligned}
W_{2}= & \rho\left\{\partial_{1}\left(\left.D F\right|_{\partial_{1} \wedge \partial_{2}}\left(\xi^{N} \wedge \partial_{2}\right)\right)+\partial_{2}\left(\left.D F\right|_{\partial_{1} \wedge \partial_{2}}\left(\partial_{1} \wedge \xi^{N}\right)\right)\right\} \\
= & \partial_{1}\left(\left.\rho D F\right|_{\partial_{1} \wedge \partial_{2}}\left(\xi^{N} \wedge \partial_{2}\right)\right)+\partial_{2}\left(\left.\rho D F\right|_{\partial_{1} \wedge \partial_{2}}\left(\partial_{1} \wedge \xi^{N}\right)\right) \\
& -\left.\sqrt{g}\left(\partial_{1} \rho\right) \frac{1}{\left|\partial_{1} \wedge \partial_{2}\right|} D F\right|_{\partial_{1} \wedge \partial_{2}}\left(\xi^{N} \wedge \partial_{2}\right)-\left.\sqrt{g}\left(\partial_{2} \rho\right) \frac{1}{\left|\partial_{1} \wedge \partial_{2}\right|} D F\right|_{\partial_{1} \wedge \partial_{2}}\left(\partial_{1} \wedge \xi^{N}\right) \\
= & \partial_{1}\left(\left.\rho D F\right|_{\partial_{1} \wedge \partial_{2}}\left(\xi^{N} \wedge \partial_{2}\right)\right)+\partial_{2}\left(\left.\rho D F\right|_{\partial_{1} \wedge \partial_{2}}\left(\partial_{1} \wedge \xi^{N}\right)\right) \\
& -\sum_{i=1}^{n-2}\left(\xi^{N} \cdot v_{i}\right) \sqrt{g} \frac{1}{\left|\partial_{1} \wedge \partial_{2}\right|}\left(\left.D F\right|_{\partial_{1} \wedge \partial_{2}}\left(\nu_{i} \wedge \partial_{2}\right)\right) \partial_{1} \rho \\
& -\sum_{i=1}^{n-2}\left(\xi^{N} \cdot v_{i}\right) \sqrt{g} \frac{1}{\left|\partial_{1} \wedge \partial_{2}\right|}\left(\left.D F\right|_{\partial_{1} \wedge \partial_{2}}\left(\partial_{1} \wedge v_{i}\right)\right) \partial_{2} \rho \\
= & \partial_{1}\left(\left.\rho D F\right|_{\partial_{1} \wedge \partial_{2}}\left(\xi^{N} \wedge \partial_{2}\right)\right)+\partial_{2}\left(\left.\rho D F\right|_{\partial_{1} \wedge \partial_{2}}\left(\partial_{1} \wedge \xi^{N}\right)\right)-\sum_{i=1}^{n-2}\left(\xi^{N} \cdot v_{i}\right) \sqrt{g} G_{v_{i}}(\rho)
\end{aligned}
$$

where $G_{v_{i}}$ is defined as follows. 
DEFINITION 4.2 Let $v$ be a unit vector field normal to $M$ and $f: M \rightarrow \mathbb{R}\left(\right.$ or $f: M \rightarrow \mathbb{R}^{n}$ ) a smooth map. We denote by $G_{v}$ the linear operator defined in local coordinates by

$$
\left.G_{v}(f):=\frac{1}{\left|\partial_{1} \wedge \partial_{2}\right|}\left(\left.D F\right|_{\partial_{1} \wedge \partial_{2}}\left(v \wedge \partial_{2}\right)\right) \partial_{1} f+\frac{1}{\left|\partial_{1} \wedge \partial_{2}\right|}\left(\left.D F\right|_{\partial_{1} \wedge \partial_{2}}\left(\partial_{1} \wedge v\right)\right)\right) \partial_{2} f
$$

In Lemma 4.2 below we show that $G_{v}$ is well defined. With the arguments used in the proof of Lemma 4.3 one can show that for any smooth function $f: M \rightarrow \mathbb{R}$ the vector $\sum_{i=1}^{n-2} G_{v_{i}}(f) v_{i}$ does not depend on the particular choice of the orthonormal frame $\left\{v_{1}, \ldots, v_{n-2}\right\}$.

In view of the expressions obtained for $W_{1}$ and $W_{2}$ we can compute

$$
\begin{aligned}
& I=\sum_{\beta \in \mathcal{I}} \int_{y \in W_{\beta}} \rho_{\beta} \circ \varphi_{\beta}\left\{\frac{\partial}{\partial y_{1}}\left(\left.D F\right|_{\partial_{1}^{\beta} \wedge \partial_{2}^{\beta}}\left(\left(\xi \circ \varphi_{\beta}\right)(y) \wedge \frac{\partial \varphi_{\beta}}{\partial y_{2}}(y)\right)\right)\right. \\
& \left.+\frac{\partial}{\partial y_{2}}\left(\left.D F\right|_{\partial_{1}^{\beta} \wedge \partial_{2}^{\beta}}\left(\frac{\partial \varphi_{\beta}}{\partial y_{1}}(y) \wedge\left(\xi \circ \varphi_{\beta}\right)(y)\right)\right)\right\} \mathrm{d} y_{1} \mathrm{~d} y_{2} \\
& =\sum_{\beta \in \mathcal{I}} \int_{y \in W_{\beta}} \rho_{\beta} \circ \varphi_{\beta} \operatorname{div}_{M}\left(F\left(\frac{\partial_{1}^{\beta} \wedge \partial_{2}^{\beta}}{\left|\partial_{1}^{\beta} \wedge \partial_{2}^{\beta}\right|}\right) \xi^{T}\right) \sqrt{g_{\beta}} \mathrm{d} y_{1} \mathrm{~d} y_{2} \\
& +\sum_{\beta \in \mathcal{I}} \int_{y \in W_{\beta}} \frac{\partial}{\partial y_{1}}\left(\left.\rho_{\beta} \circ \varphi_{\beta} D F\right|_{\partial_{1}^{\beta} \wedge \partial_{2}^{\beta}}\left(\xi^{N} \wedge \partial_{2}^{\beta}\right)\right) \mathrm{d} y_{1} \mathrm{~d} y_{2} \\
& +\sum_{\beta \in \mathcal{I}} \int_{y \in W_{\beta}} \frac{\partial}{\partial y_{2}}\left(\left.\rho_{\beta} \circ \varphi_{\beta} D F\right|_{\partial_{1}^{\beta} \wedge \partial_{2}^{\beta}}\left(\partial_{1}^{\beta} \wedge \xi^{N}\right)\right) \mathrm{d} y_{1} \mathrm{~d} y_{2} \\
& -\sum_{\beta \in \mathcal{I}} \int_{y \in W_{\beta}} \sum_{i=1}^{n-2}\left(\xi^{N} \cdot v_{i}\right) G_{\nu_{i}}\left(\rho^{\beta}\right) \sqrt{g_{\beta}} \mathrm{d} y_{1} \mathrm{~d} y_{2} \\
& =\int_{M} \operatorname{div}_{M}\left(F\left(\frac{\partial_{1} \wedge \partial_{2}}{\left|\partial_{1} \wedge \partial_{2}\right|}\right) \xi^{T}\right) \mathrm{d} A-\int_{M} \sum_{i=1}^{n-2}\left(\xi^{N} \cdot v_{i}\right) G_{v_{i}}(1) \mathrm{d} A=0,
\end{aligned}
$$

where we have used integration by parts on the domains $W_{\beta}$ together with the fact that $\rho_{\beta}$ is compactly supported, the divergence theorem (see for example [17, p. 43]), the linearity of $\sum_{i=1}^{n-2} v_{i} G_{v_{i}}(\cdot)$ and the fact that $\sum_{i=1}^{n-2} v_{i} G_{v_{i}}(1)=0$ on $M$.

Next we consider the integral $I I$. The integrand can be written (again omitting the indices $\beta$ for readability)

$$
\begin{aligned}
\rho\left(\partial_{1}\left(\left.D F\right|_{\partial_{1} \wedge \partial_{2}}\right)(\xi\right. & \left.\left.\wedge \partial_{2}\right)+\partial_{2}\left(\left.D F\right|_{\partial_{1} \wedge \partial_{2}}\right)\left(\partial_{1} \wedge \xi\right)\right) \\
& =\rho\left(\left.D^{2} F\right|_{\partial_{1} \wedge \partial_{2}}\left(\partial_{1}\left(\partial_{1} \wedge \partial_{2}\right)\right)\left(\xi \wedge \partial_{2}\right)+\left.D^{2} F\right|_{\partial_{1} \wedge \partial_{2}}\left(\partial_{2}\left(\partial_{1} \wedge \partial_{2}\right)\right)\left(\partial_{1} \wedge \xi\right)\right) \\
& =\rho\left(\left.D^{2} F\right|_{\partial_{1} \wedge \partial_{2}}\left(\partial_{1}\left(\partial_{1} \wedge \partial_{2}\right)\right)\left(\xi^{N} \wedge \partial_{2}\right)+\left.D^{2} F\right|_{\partial_{1} \wedge \partial_{2}}\left(\partial_{2}\left(\partial_{1} \wedge \partial_{2}\right)\right)\left(\partial_{1} \wedge \xi^{N}\right)\right) \\
& \quad\left(\text { since }\left.D^{2} F\right|_{\partial_{1} \wedge \partial_{2}}\left(\partial_{1} \wedge \partial_{2}\right)(\cdot)=0 \text { by (2.3) }\right) \\
& =\rho \sqrt{g} \sum_{i=1}^{n-2}\left(\xi^{N} \cdot v_{i}\right) \frac{1}{\left|\partial_{1} \wedge \partial_{2}\right|}\left(\partial_{1}\left(\left.D F\right|_{\partial_{1} \wedge \partial_{2}}\right)\left(v_{i} \wedge \partial_{2}\right)+\partial_{2}\left(\left.D F\right|_{\partial_{1} \wedge \partial_{2}}\right)\left(\partial_{1} \wedge v_{i}\right)\right) \\
& =\rho \sqrt{g} \xi^{N} \cdot H_{F},
\end{aligned}
$$

where $H_{F}$ is the anisotropic mean curvature vector described in Definition 4.1 
Thus we can write

$$
\begin{aligned}
I I= & -\sum_{\beta \in \mathcal{I}} \int_{y \in W_{\beta}} \rho_{\beta} \circ \varphi_{\beta}\left\{\frac { \partial } { \partial y _ { 1 } } \left(\left.D F\right|_{\partial_{1}^{\beta} \wedge \partial_{2}^{\beta}}\left(\left(\xi \circ \varphi_{\beta}\right)(y) \wedge \frac{\partial \varphi_{\beta}}{\partial y_{2}}(y)\right)\right.\right. \\
& +\frac{\partial}{\partial y_{2}}\left(\left.D F\right|_{\left.\partial_{1}^{\beta} \wedge \partial_{2}^{\beta}\right)}\left(\frac{\partial \varphi_{\beta}}{\partial y_{1}}(y) \wedge\left(\xi \circ \varphi_{\beta}\right)(y)\right)\right\} \mathrm{d} y_{1} \mathrm{~d} y_{2} \\
= & -\sum_{\beta \in \mathcal{I}} \int_{y \in W_{\beta}} \rho_{\beta} \circ \varphi_{\beta} \xi^{N} \cdot H_{F} \sqrt{g_{\beta}} \mathrm{d} y_{1} \mathrm{~d} y_{2}=-\int_{M} \xi^{N} \cdot H_{F} \mathrm{~d} A .
\end{aligned}
$$

Putting all estimates together we conclude that

$$
\left.\frac{\mathrm{d}}{\mathrm{d} t}\right|_{t=0}\left|M^{t}\right|_{F}=I+I I=-\int_{M} \xi^{N} \cdot H_{F} \mathrm{~d} A=-\int_{M} \xi \cdot H_{F} \mathrm{~d} A .
$$

Before giving a definition of motion by anisotropic mean curvature, we give a few lemmas, in which we prove that Definitions 4.1 and 4.2 , naturally arising during the computation of the first variation, are correct. Moreover, we show that we can retrieve well known results for the special case of $n=3$ and for the isotropic setting.

Lemma 4.2 Let $v$ be a unit vector field normal to $M$ and $f: M \rightarrow \mathbb{R}\left(\right.$ or $f: M \rightarrow \mathbb{R}^{n}$ ) a smooth map. The linear operator $G_{\nu}$, whose description in local coordinates is given in 4.10 , is well defined.

Proof. We need to show that the expression (4.10) does not depend on the choice of the coordinate functions. At $x \in M$, let $\tilde{\partial}_{1}, \tilde{\partial}_{2}$ be another basis for the tangent space $T_{x} M$, equioriented with $\left\{\partial_{1}, \partial_{2}\right\}$. We can write

$$
\begin{aligned}
& \partial_{1}=a \tilde{\partial}_{1}+b \tilde{\partial}_{2}, \\
& \partial_{2}=c \tilde{\partial}_{1}+d \tilde{\partial}_{2},
\end{aligned}
$$

for appropriate coefficients $a, b, c, d$. Setting $A:=\left(\begin{array}{ll}a & b \\ c & d\end{array}\right)$, we immediately infer that $\partial_{1} \wedge \partial_{2}=$ $\operatorname{det}(A) \tilde{\partial}_{1} \wedge \tilde{\partial}_{2}$, with $\operatorname{det}(A)>0$ due to the equiorientation. From (2.4) we infer that $\left.D F\right|_{\partial_{1} \wedge \partial_{2}}=$ $\left.D F\right|_{\tilde{\partial}_{1} \wedge \tilde{\partial}_{2}}$. Therefore we obtain

$$
\begin{aligned}
\left(\left.D F\right|_{\partial_{1} \wedge \partial_{2}}\left(\nu \wedge \partial_{2}\right)\right) \partial_{1} f+ & \left(\left.D F\right|_{\partial_{1} \wedge \partial_{2}}\left(\partial_{1} \wedge v\right)\right) \partial_{2} f \\
= & \left(\left.D F\right|_{\tilde{\partial}_{1} \wedge \tilde{\partial}_{2}}\left(v \wedge c \tilde{\partial}_{1}+d \tilde{\partial}_{2}\right)\right)\left(a \tilde{\partial}_{1}+b \tilde{\partial}_{2}\right)(f) \\
& +\left(\left.D F\right|_{\tilde{\partial}_{1} \wedge \tilde{\partial}_{2}}\left(a \tilde{\partial}_{1}+b \tilde{\partial}_{2} \wedge v\right)\right)\left(c \tilde{\partial}_{1}+d \tilde{\partial}_{2}\right)(f) \\
= & \left.\operatorname{det}(A) D F\right|_{\tilde{1}_{1} \wedge \tilde{\partial}_{2}}\left(\tilde{\partial}_{1} \wedge v\right) \tilde{\partial}_{2} f+\left.\operatorname{det}(A) D F\right|_{\tilde{\partial}_{1} \wedge \tilde{\partial}_{2}}\left(v \wedge \tilde{\partial}_{2}\right) \tilde{\partial}_{1} f,
\end{aligned}
$$

and the claim follows immediately.

LEMMA 4.3 The anisotropic mean curvature vector $H_{F}$, whose description in local coordinates is given in 4.4, is well defined.

Proof. We need to show that the expression in 4.4 is independent of the choice of the local coordinates and the orthonormal frame $\left\{v_{1}, \ldots, v_{n-2}\right\}$ normal to $M$. 
The first claim is showed by employing the same sort of argument used in the proof of Lemma 4.2, thus we do not report it here.

Next we show that the definition of $H_{F}$ does not depend on the choice of the orthonormal frame. Let $\left\{\tilde{v}_{1}, \ldots, \tilde{v}_{n-2}\right\}$ be another orthonormal frame. We can write $v_{i}=\sum_{k=1}^{n-2} b_{i k} \tilde{v}_{k}$. The matrix $B=\left(b_{i j}\right)_{i, j=1, \ldots, n-2}$ is orthogonal and thus $\sum_{i=1}^{n-2} b_{i k} b_{i l}=\delta_{k l}$. We have

$$
\begin{aligned}
& \sum_{i=1}^{n-2}\left(\partial_{1}\left(\left.D F\right|_{\partial_{1} \wedge \partial_{2}}\right)\left(v_{i} \wedge \partial_{2}\right)+\partial_{2}\left(\left.D F\right|_{\partial_{1} \wedge \partial_{2}}\right)\left(\partial_{1} \wedge v_{i}\right)\right) v_{i} \\
& =\sum_{i=1}^{n-2}\left(\partial_{1}\left(\left.D F\right|_{\partial_{1} \wedge \partial_{2}}\right)\left(\sum_{k=1}^{n-2} b_{i k} \tilde{v}_{k} \wedge \partial_{2}\right)+\partial_{2}\left(\left.D F\right|_{\partial_{1} \wedge \partial_{2}}\right)\left(\partial_{1} \wedge \sum_{j=1}^{n-2} b_{i j} \tilde{v}_{j}\right)\right) \sum_{l=1}^{n-2} b_{i l} \tilde{v}_{l} \\
& =\sum_{i, k, l=1}^{n-2} b_{i k} b_{i l} \partial_{1}\left(\left.D F\right|_{\partial_{1} \wedge \partial_{2}}\right)\left(\tilde{v}_{k} \wedge \partial_{2}\right) \tilde{v}_{l}+\sum_{i, j, l=1}^{n-2} b_{i j} b_{i l} \partial_{2}\left(\left.D F\right|_{\partial_{1} \wedge \partial_{2}}\right)\left(\partial_{1} \wedge \tilde{v}_{j}\right) \tilde{v}_{l} \\
& =\sum_{i=1}^{n-2}\left(\partial_{1}\left(\left.D F\right|_{\partial_{1} \wedge \partial_{2}}\right)\left(\tilde{v}_{i} \wedge \partial_{2}\right)+\partial_{2}\left(\left.D F\right|_{\partial_{1} \wedge \partial_{2}}\right)\left(\partial_{1} \wedge \tilde{v}_{i}\right)\right) \tilde{v}_{i},
\end{aligned}
$$

and the claim follows.

REMARK 4.4 If the anisotropy function $F: \Lambda^{2} \mathbb{R}^{n} \rightarrow \mathbb{R}$ is reversible then the anisotropy vector $H_{F}$ is well defined even if the surface $M$ is not orientable.

One uses the fact that for $\lambda<0$ we have $F\left(\lambda p_{1} \wedge p_{2}\right)=-\lambda F\left(p_{1} \wedge p_{2}\right)$ and thus $\left.D F\right|_{\lambda p_{1} \wedge p_{2}}=$ $-\left.D F\right|_{p_{1} \wedge p_{2}}$ and $\left.D^{2} F\right|_{\lambda p_{1} \wedge p_{2}}=-\left.(1 / \lambda) D^{2} F\right|_{p_{1} \wedge p_{2}}$.

Similarly, the expression 2.1 does not depend on the orientation chosen for the (connected) manifold $M$ provided $F$ is a norm.

REMARK 4.5 In the isotropic case $H_{F}$ coincides with the classical mean curvature vector $H$.

Note that as in [7], [6], and [9], the mean curvature vector here is defined as $H=\sum_{i=1}^{n-2} \operatorname{Tr} S_{v_{i}} v_{i}$ where $\left\{v_{1}, \ldots, v_{n-2}\right\}$ is an orthonormal frame normal to $M$, and $S$ denotes the classical shape operator (as defined in [11, Chapter 6]). This definition differs from the more common definition $H=\frac{1}{2} \sum_{i=1}^{n-2} \operatorname{Tr} S_{\nu_{i}} \nu_{i}$.

Let us consider the isotropic case, where $F\left(p_{1} \wedge p_{2}\right)=\left|p_{1} \wedge p_{2}\right|$. Here we observe that $\left.D F\right|_{p_{1} \wedge p_{2}}(\cdot)=\left\langle p_{1} \wedge p_{2} /\left|p_{1} \wedge p_{2}\right|, \cdot\right\rangle$. To compute $H_{F}$ at $x \in M$ we can assume that $\partial_{i} \cdot \partial_{j}=\delta_{i j}$ at $x \in M$ (for the existence of normal coordinates see for instance [13, §2.100]), and write

$$
\begin{aligned}
H_{F}= & \sum_{i=1}^{n-2} \frac{1}{\left|\partial_{1} \wedge \partial_{2}\right|}\left(\partial_{1}\left(\left.D F\right|_{\partial_{1} \wedge \partial_{2}}\right)\left(v_{i} \wedge \partial_{2}\right)+\partial_{2}\left(\left.D F\right|_{\partial_{1} \wedge \partial_{2}}\right)\left(\partial_{1} \wedge v_{i}\right)\right) v_{i} \\
= & \sum_{i=1}^{n-2}\left(\left\langle\partial_{1}\left(\partial_{1} \wedge \partial_{2}\right), v_{i} \wedge \partial_{2}\right\rangle+\left\langle\partial_{2}\left(\partial_{1} \wedge \partial_{2}\right), \partial_{1} \wedge v_{i}\right\rangle\right) v_{i} \\
= & \sum_{i=1}^{n-2}\left(\left\langle\bar{\nabla}_{\partial_{1}} \partial_{1} \wedge \partial_{2}, v_{i} \wedge \partial_{2}\right\rangle+\left\langle\partial_{1} \wedge \bar{\nabla}_{\partial_{1}} \partial_{2}, v_{i} \wedge \partial_{2}\right\rangle\right. \\
& \left.+\left\langle\bar{\nabla}_{\partial_{2}} \partial_{1} \wedge \partial_{2}, \partial_{1} \wedge v_{i}\right\rangle+\left\langle\partial_{1} \wedge \bar{\nabla}_{\partial_{2}} \partial_{2}, \partial_{1} \wedge v_{i}\right\rangle\right) v_{i}
\end{aligned}
$$

(where $\bar{\nabla}$ denotes the Levi-Civita connection on $\mathbb{R}^{n}$ ) 
$=\sum_{i=1}^{n-2}\left(\bar{\nabla}_{\partial_{1}} \partial_{1} \cdot v_{i}+\bar{\nabla}_{\partial_{2}} \partial_{2} \cdot v_{i}\right) v_{i}$

(by (3.1) and the orthonormality of $\left\{\partial_{1}, \partial_{2}, v_{1}, \ldots, v_{n-2}\right\}$ at $x$ )

$$
\begin{aligned}
& =\sum_{i=1}^{n-2}\left(-\bar{\nabla}_{\partial_{1}} v_{i} \cdot \partial_{1}-\bar{\nabla}_{\partial_{2}} \nu_{i} \cdot \partial_{2}\right) v_{i}=\sum_{i=1}^{n-2}\left(-\left(\bar{\nabla}_{\partial_{1}} v_{i}\right)^{T} \cdot \partial_{1}-\left(\bar{\nabla}_{\partial_{2}} \nu_{i}\right)^{T} \cdot \partial_{2}\right) v_{i} \\
& =\sum_{i=1}^{n-2}\left(S_{\nu_{i}}\left(\partial_{1}\right) \cdot \partial_{1}+S_{\nu_{i}}\left(\partial_{2}\right) \cdot \partial_{2}\right) v_{i}=\sum_{i=1}^{n-2} \operatorname{Tr} S_{\nu_{i}} v_{i}=H .
\end{aligned}
$$

REMARK 4.6 For $n=3$, i.e.in the case where the codimension is one, we retrieve the result which appeared in [6].

At a point $x \in M$ consider a coordinate system such that $\partial_{1}, \partial_{2}$ are orthonormal at $x$, and choose the unit normal $v$ so that $\left\{\partial_{1}, \partial_{2}, v\right\}$ is equioriented with the canonical basis $\left\{e_{1}, e_{2}, e_{3}\right\}$, i.e. $\partial_{1} \wedge \partial_{2} \wedge v=e_{1} \wedge e_{2} \wedge e_{3}$.

As in Section 3 define $\tilde{F}: \mathbb{R}^{3} \rightarrow \mathbb{R}$ by $\tilde{F}=F \circ *$, where $*$ is the Hodge operator. Recall that $*\left(\partial_{1} \wedge \partial_{2}\right)=v, *\left(\partial_{1} \wedge v\right)=-\partial_{2}, *\left(v \wedge \partial_{2}\right)=-\partial_{1}, * *\left(p_{1} \wedge p_{2}\right)=p_{1} \wedge p_{2}$, and $\left\langle p_{1} \wedge p_{2}, q_{1} \wedge q_{2}\right\rangle=$ $*\left(p_{1} \wedge p_{2}\right) \cdot *\left(q_{1} \wedge q_{2}\right)$. From

$$
\begin{aligned}
\nabla \tilde{F}(\nu) \cdot w & =\left.D \tilde{F}\right|_{\nu}(w)=\left.D F\right|_{* \nu}(* w)=\left.D F\right|_{\partial_{1} \wedge \partial_{2}}(* w) \\
& =\left\langle\nabla F\left(\partial_{1} \wedge \partial_{2}\right), * w\right\rangle=* \nabla F\left(\partial_{1} \wedge \partial_{2}\right) \cdot w
\end{aligned}
$$

for all $w \in \mathbb{R}^{3}$, we infer that $* \nabla F\left(\partial_{1} \wedge \partial_{2}\right)=\nabla \tilde{F}(\nu)$. Thus at $x \in M$ we can compute

$$
\begin{aligned}
H_{F} & =\left(\partial_{1}\left(\left.D F\right|_{\partial_{1} \wedge \partial_{2}}\right)\left(v \wedge \partial_{2}\right)+\partial_{2}\left(\left.D F\right|_{\partial_{1} \wedge \partial_{2}}\right)\left(\partial_{1} \wedge v\right)\right) v \\
& =\left(\left\langle\partial_{1}\left(\nabla F\left(\partial_{1} \wedge \partial_{2}\right)\right), v \wedge \partial_{2}\right\rangle+\left\langle\partial_{2}\left(\nabla F\left(\partial_{1} \wedge \partial_{2}\right)\right), \partial_{1} \wedge v\right\rangle\right) v \\
& =\left(* \partial_{1}\left(\nabla F\left(\partial_{1} \wedge \partial_{2}\right)\right) \cdot *\left(v \wedge \partial_{2}\right)+* \partial_{2}\left(\nabla F\left(\partial_{1} \wedge \partial_{2}\right)\right) \cdot *\left(\partial_{1} \wedge v\right)\right) v \\
& =\left(-\partial_{1} \nabla \tilde{F}(v) \cdot \partial_{1}-\partial_{2} \nabla \tilde{F}(v) \cdot \partial_{2}\right) v=-\operatorname{div}_{M}(\nabla \tilde{F}(v)) v .
\end{aligned}
$$

(A definition of divergence for not necessarily tangential vector fields can be found in [17] and [7].) Therefore we infer

$$
\left.\frac{\mathrm{d}}{\mathrm{d} t}\right|_{t=0}\left|M^{t}\right|_{F}=-\int_{M} \xi \cdot H_{F} \mathrm{~d} A=\int_{M} \xi \cdot \operatorname{div}_{M}(\nabla \tilde{F}(v)) v \mathrm{~d} A .
$$

This is the same result presented in [6]. Note that in the proof given in [6] the fact that the codimension is equal to one plays a fundamental role, whereas with our approach this is no longer the case.

We are now able to give the definition of anisotropic mean curvature flow.

Definition 4.3 Let $F: \Lambda^{2} \mathbb{R}^{n} \rightarrow[0, \infty)$ be a reversible anisotropic function and $\mathbf{x}: \tilde{M} \rightarrow \mathbb{R}^{n}$ be an isometric immersion of a smooth compact two-dimensional submanifold. The anisotropic mean curvature flow is a family of immersions $\mathbf{x}:[0, T) \times \tilde{M} \rightarrow \mathbb{R}^{n}$ parametrized by $t$ that satisfies

$$
\left\{\begin{array}{l}
\mathbf{x}_{t}(t, y)=H_{F}(t, y) \\
\mathbf{x}(0)=\mathbf{x}
\end{array}\right.
$$

where $H_{F}(t, y)$ is the anisotropic mean curvature vector of $\mathbf{x}(t, \tilde{M})=: M(t)$ at $\mathbf{x}(t, y)$. 
Observe that $M(t)$ is an immersed surface and so may have self-intersections. However, since immersions are locally embeddings, the anisotropic mean curvature vector is well defined.

For non-reversible anisotropy functions $F$, one can modify the above definition by taking $\tilde{M}$ to be orientable and by considering a family of oriented immersions.

Apart from the results described in [3] for a volume-preserving mean curvature flow and classical results for the isotropic mean curvature flow (see for example [22] and references given in there), the author is not aware of the existence of proofs of short and long time existence for the anisotropic mean curvature flow.

\section{A weak formulation of the AMCF}

Our goal is to give a finite element discretization of the anisotropic mean curvature flow (AMCF). Since we would like to use piecewise linear elements, we should first of all obtain a description of the flow which does not contain derivatives of second order of the coordinate maps. Moreover, in order to allow for numerical experiments with crystalline norms, we would like to avoid having second derivatives of the anisotropy function $F$.

To derive a weak formulation for the flow that suits our needs, let us have a look again at $\left.\frac{\mathrm{d}}{\mathrm{d} t}\right|_{t=0}\left|M^{t}\right|_{F}$ as considered in Section 4

Omitting the indices $\beta$ relating to the coordinate charts, taking $\left\{v_{1}, \ldots, v_{n-2}\right\}$ to be an orthonormal frame normal to $M$, and recalling Definition 4.2 and 2.3 , we can write the expression (4.8) as

$$
\begin{aligned}
\left.\frac{\mathrm{d}}{\mathrm{d} t}\right|_{t=0} F\left(\partial_{1}^{t} \wedge \partial_{2}^{t}\right)= & \left.D F\right|_{\partial_{1} \wedge \partial_{2}}\left(\partial_{1} \xi \wedge \partial_{2}+\partial_{1} \wedge \partial_{2} \xi\right) \\
= & \left.D F\right|_{\partial_{1} \wedge \partial_{2}}\left(\left(\left(\partial_{1} \xi\right)_{1}^{T} \partial_{1}+\left(\partial_{1} \xi\right)_{2}^{T} \partial_{2}+\sum_{i=1}^{n-2} \partial_{1} \xi \cdot v_{i} v_{i}\right) \wedge \partial_{2}\right) \\
& +\left.D F\right|_{\partial_{1} \wedge \partial_{2}}\left(\partial_{1} \wedge\left(\left(\partial_{2} \xi\right)_{1}^{T} \partial_{1}+\left(\partial_{2} \xi\right)_{2}^{T} \partial_{2}+\sum_{i=1}^{n-2} \partial_{2} \xi \cdot v_{i} \nu_{i}\right)\right) \\
= & \left.\left(\partial_{1} \xi\right)_{1}^{T} D F\right|_{\partial_{1} \wedge \partial_{2}}\left(\partial_{1} \wedge \partial_{2}\right)+\left.\sum_{i=1}^{n-2}\left(\partial_{1} \xi \cdot v_{i}\right) D F\right|_{\partial_{1} \wedge \partial_{2}}\left(v_{i} \wedge \partial_{2}\right) \\
& +\left.\left(\partial_{2} \xi\right)_{2}^{T} D F\right|_{\partial_{1} \wedge \partial_{2}}\left(\partial_{1} \wedge \partial_{2}\right)+\left.\sum_{i=1}^{n-2}\left(\partial_{2} \xi \cdot v_{i}\right) D F\right|_{\partial_{1} \wedge \partial_{2}}\left(\partial_{1} \wedge v_{i}\right) \\
= & F\left(\partial_{1} \wedge \partial_{2}\right)\left(\left(\partial_{1} \xi\right)_{1}^{T}+\left(\partial_{2} \xi\right)_{2}^{T}\right)+\sum_{i=1}^{n-2} G_{v_{i}}(\xi) \cdot v_{i} \sqrt{g} \\
= & F\left(\frac{\partial_{1} \wedge \partial_{2}}{\left|\partial_{1} \wedge \partial_{2}\right|}\right) \nabla_{M} \xi \cdot \nabla_{M} \operatorname{Id} \sqrt{g}+\sum_{i=1}^{n-2} G_{v_{i}}(\xi) \cdot v_{i} \sqrt{g} .
\end{aligned}
$$

In the last equality we have used the fact that

$$
\begin{aligned}
\left(\partial_{1} \xi\right)_{1}^{T}+\left(\partial_{2} \xi\right)_{2}^{T} & =\sum_{j=1}^{2} g^{1 j} \partial_{1} \xi \cdot \partial_{j}+\sum_{j=1}^{2} g^{2 j} \partial_{2} \xi \cdot \partial_{j}=\sum_{i, j=1}^{2} g^{i j} \partial_{i} \xi \cdot \partial_{j} \\
& =\sum_{i=1}^{n} \nabla_{M} \xi_{i} \cdot \nabla_{M}(\mathrm{Id})_{i}=\nabla_{M} \xi \cdot \nabla_{M} \mathrm{Id}
\end{aligned}
$$


where $\nabla_{M}$ denotes the tangential gradient and as usual $\left(g^{i j}\right)_{i, j=1,2}$ is such that $\sum_{k} g^{i k} g_{k j}=\delta_{i j}$, where $g_{i j}=\partial_{i} \cdot \partial_{j}$. Recall also that the map $\sum_{i=1}^{n-2} G_{v_{i}}(\xi) \cdot v_{i}$ does not depend on the choice of the orthonormal frame $\left\{v_{1}, \ldots, v_{n-2}\right\}$.

The advantage of the expression obtained above is that it is coordinate free and satisfies the requirements mentioned above. In particular, we can write

$$
\begin{aligned}
\left.\frac{\mathrm{d}}{\mathrm{d} t}\right|_{t=0}\left|M^{t}\right|_{F} & =\left.\sum_{\beta \in \mathcal{I}} \int_{y \in W_{\beta}} \rho_{\beta} \circ \varphi_{\beta} \frac{\mathrm{d}}{\mathrm{d} t}\right|_{t=0} F\left(\partial_{1}^{\beta, t} \wedge \partial_{2}^{\beta, t}\right) \mathrm{d} y_{1} \mathrm{~d} y_{2} \\
& =\int_{M} F\left(\frac{\partial_{1} \wedge \partial_{2}}{\left|\partial_{1} \wedge \partial_{2}\right|}\right) \nabla_{M} \mathrm{Id} \cdot \nabla_{M} \xi \mathrm{d} A+\int_{M} \sum_{i=1}^{n-2} G_{v_{i}}(\xi) \cdot v_{i} \mathrm{~d} A .
\end{aligned}
$$

Note that in the isotropic case the last integral vanishes.

We now give a formulation of the anisotropic mean curvature flow which we will be able to discretize by using piecewise linear finite elements.

Definition 5.1 (Weak formulation for the AMCF) Let $F, \tilde{M}, \mathbf{x}, M(t)$ be as in Definition 4.3 . We look for immersions $\mathbf{x}(t, \cdot): \tilde{M} \rightarrow \mathbb{R}^{n}, t \in[0, T)$, such that $\mathbf{x}(0)=\mathbf{x}$ and

$$
\int_{\tilde{M}} \partial_{t} \mathbf{x} \cdot \tilde{\xi} \mathrm{d} V(t)+\int_{\tilde{M}} F\left(\frac{\partial_{1} \wedge \partial_{2}}{\left|\partial_{1} \wedge \partial_{2}\right|}\right) \nabla_{\tilde{M}} \mathbf{x} \cdot \nabla_{\tilde{M}} \tilde{\xi} \mathrm{d} V(t)=-\int_{\tilde{M}} \sum_{i=1}^{n-2} G_{v_{i}}(\xi) \cdot v_{i} \mathrm{~d} V(t)
$$

for every $\tilde{\xi} \in C_{0}^{1}\left(\tilde{M}, \mathbb{R}^{n}\right), \xi: M(t) \rightarrow \mathbb{R}^{n}$ such that $\tilde{\xi}=\xi \circ \mathbf{x}(t)$, and for $\left\{v_{1}, \ldots, v_{n-2}\right\}$ orthonormal frame normal to $M(t)$. We use the notation $\mathrm{d} V(t)$ for the volume element induced by the isometric immersion $\mathbf{x}(t)$ and $\partial_{i}=D \mathbf{x}(t)\left(e_{i}\right)$ for $e_{i} \in T, \tilde{M}, i=1,2$.

Note also that when we write $\nabla_{\tilde{M}} \mathbf{x} \cdot \nabla_{\tilde{M}} \tilde{\xi}$ we mean $\sum_{i=1}^{n} g\left(\nabla_{\tilde{M}} \mathbf{x}_{i}, \nabla_{\tilde{M}} \tilde{\xi}_{i}\right)$, where $g(\cdot, \cdot)$ is the metric on $\tilde{M}$ induced by the isometric immersion $\mathbf{x}(t)$, i.e. $g(v, w)=D \mathbf{x}(t)(v) \cdot D \mathbf{x}(t)(w)$ for all $v, w \in T . \tilde{M}$.

For the special case of $n=3$, we recover the weak formulation presented in [7, \$4.2].

In our applications, $\tilde{M}$ will typically be a closed compact surface embedded in $\mathbb{R}^{n}$ through the inclusion map (coordinate map) $\mathbf{x}$.

\section{Discretization}

For the discretization we first propose the following scheme, which for the special case of $n=3$ coincides with the one described in [7, \$4.2].

In the following, $f^{(k)}$ stands for the evaluation of a generic function $f$ at the $k$-th time level. Furthermore, we denote by $\tau$ the time step and by $M^{(0)}=\mathbf{x}^{(0)}(\tilde{M})$ the initial surface.

In each time step we view $\mathbf{x}^{(k+1)}$ as a map from $M^{(k)}$ to $M^{(k+1)}$. The time discretization is described by

$$
\begin{aligned}
\int_{M^{(k)}} \frac{1}{\tau}\left(\mathbf{x}^{(k+1)}-\mathrm{Id}\right) \cdot \xi \mathrm{d} A+\int_{M^{(k)}} F\left(\frac{\partial_{1}^{(k)} \wedge \partial_{2}^{(k)}}{\left|\partial_{1}^{(k)} \wedge \partial_{2}^{(k)}\right|}\right) & \nabla_{M^{(k)}} \mathbf{x}^{(k+1)} \cdot \nabla_{M^{(k)}} \xi \mathrm{d} A \\
& =-\int_{M^{(k)}} \sum_{i=1}^{n-2} G_{v_{i}^{(k)}}^{(k)}(\xi) \cdot v_{i}^{(k)} \mathrm{d} A
\end{aligned}
$$


for any $\xi \in C_{0}^{1}\left(M^{(k)}, \mathbb{R}^{n}\right)$; recall that in local coordinates

$$
\begin{aligned}
G_{v_{i}^{(k)}}^{(k)}(\xi)=\frac{1}{\left|\partial_{1}^{(k)} \wedge \partial_{2}^{(k)}\right|}\left(D F | _ { \partial _ { 1 } ^ { ( k ) } \wedge \partial _ { 2 } ^ { ( k ) } } \left(v_{i}^{(k)} \wedge\right.\right. & \left.\left.\partial_{2}^{(k)}\right)\right) \partial_{1}^{(k)} \xi \\
& +\frac{1}{\left|\partial_{1}^{(k)} \wedge \partial_{2}^{(k)}\right|}\left(\left.D F\right|_{\partial_{1}^{(k)} \wedge \partial_{2}^{(k)}}\left(\partial_{1}^{(k)} \wedge v_{i}^{(k)}\right)\right) \partial_{2}^{(k)} \xi
\end{aligned}
$$

for $\left\{v_{1}^{(k)}, \ldots, v_{n-2}^{(k)}\right\}$ an orthonormal frame normal to $M^{(k)}$.

The above scheme is motivated by a semi-implicit time discretization of 5.1] which treats the non-linearity in an explicit way: more precisely, consider

$$
\begin{array}{r}
\int_{\tilde{M}} \frac{1}{\tau}\left(\hat{\mathbf{x}}^{(k+1)}-\hat{\mathbf{x}}^{(k)}\right) \cdot \tilde{\xi} \mathrm{d} V(k)+\int_{\tilde{M}} F\left(\frac{\partial_{1}^{(k)} \wedge \partial_{2}^{(k)}}{\left|\partial_{1}^{(k)} \wedge \partial_{2}^{(k)}\right|}\right) \nabla_{\tilde{M}} \hat{\mathbf{x}}^{(k+1)} \cdot \nabla_{\tilde{M}} \tilde{\xi} \mathrm{d} V(k) \\
=-\int_{\tilde{M}} \sum_{i=1}^{n-2} G_{v_{i}^{(k)}}^{(k)}(\xi) \cdot v_{i}^{(k)} \mathrm{d} V(k)
\end{array}
$$

and let $M^{(k)}=\hat{\mathbf{x}}^{(k)}(\tilde{M}), \hat{\mathbf{x}}^{(k+1)}(p)=\mathbf{x}^{(k+1)}\left(\hat{\mathbf{x}}^{(k)}(p)\right)$ for $p \in \tilde{M}, \partial_{i}^{(k)}=D \hat{\mathbf{x}}^{(k)}\left(e_{i}\right)$ for $e_{i} \in T \tilde{M}$, $i=1,2$, and $\tilde{\xi}=\xi \circ \hat{\mathbf{x}}^{(k)}$.

For a spatial discretization, we first omit the time index (for readability) and denote by $M_{h}$ a polyhedron consisting of triangles and approximating $M$. As in [7] we consider the finite element space of affine linear functions on the triangles $T$ of $M_{h}$,

$$
\mathcal{V}_{h}=\left\{\Phi \in C^{0}\left(M_{h}\right): \Phi_{T} \in \mathcal{P}_{1}, T \in M_{h}\right\}
$$

The space $\mathcal{V}_{h}$ has the basis $\left\{\Phi_{j}\right\}_{j=1}^{J}$, where $J$ is the number of vertices of $M_{h}$ and $\Phi_{j}\left(X_{i}\right)=\delta_{i j}$ for all vertices $X_{i}$. In each time step we look for a solution $X \in\left[\mathcal{V}_{h}\right]^{n}$ of the form

$$
X=\sum_{j=1}^{J} \tilde{X}_{j} \Phi_{j}
$$

with $\tilde{X}_{j} \in \mathbb{R}^{n}$. We use the notation $\bar{X}_{l}=\left(\tilde{X}_{1, l}, \ldots, \tilde{X}_{J, l}\right) \in \mathbb{R}^{J}, l=1, \ldots, n$.

We propose the following scheme.

Algorithm 6.1 Let $M_{h}^{(0)}$ be an initial polyhedron interpolating the initial surface $\mathbf{x}^{(0)}(\tilde{M})$. We compute a sequence of time step solutions $X^{(k)}$ for $k=1,2, \ldots$ such that for any $\Phi \in\left[\mathcal{V}_{h}\right]^{n}$,

$$
\begin{array}{r}
\int_{M_{h}^{(k)}} \frac{1}{\tau}\left(X^{(k+1)}-\mathrm{Id}\right) \cdot \Phi \mathrm{d} A+\int_{M_{h}^{(k)}} F\left(\frac{\partial_{1}^{(k)} \wedge \partial_{2}^{(k)}}{\left|\partial_{1}^{(k)} \wedge \partial_{2}^{(k)}\right|}\right) \nabla_{M_{h}^{(k)}} X^{(k+1)} \cdot \nabla_{M_{h}^{(k)}} \Phi \mathrm{d} A \\
=-\int_{M_{h}^{(k)}} \sum_{i=1}^{n-2} G_{N_{i}^{(k)}}^{(k)}(\Phi) \cdot N_{i}^{(k)} \mathrm{d} A,
\end{array}
$$

where $\left\{N_{i}^{(k)}\right\}_{i=1}^{n-2}$ denotes the orthonormal frame of piecewise constant discrete normals to $M_{h}^{(k)}$. The next discrete surface is generated by setting

$$
M_{h}^{(k+1)}=X^{(k+1)}\left(M_{h}^{(k)}\right) .
$$


Note that the tangential gradient $\nabla_{M_{h}^{(k)}}(\cdot)$ is given piecewise on each face of $M_{h}^{(k)}$. For a given discrete surface $M_{h}^{(k)}$ we define the (symmetric) mass matrix

$$
A_{i j}^{(k)}=\int_{M_{h}^{(k)}} \Phi_{i} \Phi_{j} \mathrm{~d} A, \quad i, j=1, \ldots, J
$$

the (symmetric) stiffness matrix

$$
L_{i j}^{(k)}=\int_{M_{h}^{(k)}} F\left(\frac{\partial_{1}^{(k)} \wedge \partial_{2}^{(k)}}{\left|\partial_{1}^{(k)} \wedge \partial_{2}^{(k)}\right|}\right) \nabla_{M_{h}^{(k)}} \Phi_{i} \nabla_{M_{h}^{(k)}} \Phi_{j} \mathrm{~d} A
$$

$(i, j=1, \ldots, J)$, as well as the right-hand side

$$
\begin{aligned}
B_{l, j}^{(k)} & =\int_{M_{h}^{(k)}} x_{l} \Phi_{j} \mathrm{~d} A, \\
C_{l, j}^{(k)} & =-\int_{M_{h}^{(k)}} \sum_{i=1}^{n-2} G_{N_{i}^{(k)}}^{(k)}\left(\Phi_{j}\right)\left(N_{i}^{(k)}\right)_{l} \mathrm{~d} A,
\end{aligned}
$$

where $j=1, \ldots, J$ and $l=1, \ldots, n$.

In every time step we need to solve $n$ linear systems with the same matrix for the computation of (the spatial components) $\bar{X}_{l}^{(k+1)} \in \mathbb{R}^{J}$, namely

$$
\left(\frac{1}{\tau} A^{(k)}+L^{(k)}\right) \bar{X}_{l}^{(k+1)}=\frac{1}{\tau} B_{l}^{(k)}+C_{l}^{(k)} .
$$

Since the matrix $\frac{1}{\tau} A^{(k)}+L^{(k)}$ is symmetric and positive definite the above systems can be solved.

\subsection{The isotropic case}

In the isotropic case, Algorithm 6.1 reduces to

AlGORITHM 6.2 (Isotropic case) Given an initial discrete surface $M_{h}^{(0)}$, compute a sequence of time step solutions $X^{(k)}, k=1,2, \ldots$, such that

$$
\int_{M_{h}^{(k)}} \frac{1}{\tau}\left(X^{(k+1)}-\mathrm{Id}\right) \cdot \Phi \mathrm{d} A+\int_{M_{h}^{(k)}} \nabla_{M_{h}^{(k)}} X^{(k+1)} \cdot \nabla_{M_{h}^{(k)}} \Phi \mathrm{d} A=0
$$

for all $\Phi \in\left[\mathcal{V}_{h}\right]^{n}$. The new surface is obtained by setting $M_{h}^{(k+1)}=X^{(k+1)}\left(M_{h}^{(k)}\right)$.

In particular we recover the scheme presented in [9, §4]. This scheme has the nice property of being stable, i.e.

$$
\left|M_{h}^{(0)}\right| \geqslant \sum_{k=0}^{K} \int_{M_{h}^{(k)}} \frac{\left|X^{(k+1)}-\mathrm{Id}\right|^{2}}{\tau} \mathrm{d} A+\left|M_{h}^{(K+1)}\right| .
$$

Stability follows by testing Algorithm 6.2 with $\Phi=X^{(k+1)}-\mathrm{Id}$ and by adapting to our present situation the following results proved by Bänsch in [4]. 
Lemma 6.1 Let $m=1$ or $m=2$ and $\Gamma$ an $m$-dimensional, closed, regular $C^{0,1}$-manifold embedded in $\mathbb{R}^{k}, k \in \mathbb{N}$. Moreover let $X: \Gamma \rightarrow \operatorname{rg}(\Gamma) \subset \mathbb{R}^{k}$ be a homeomorphism with $D X,(D X)^{-1} \in L^{\infty}$. Then

$$
\int_{\Gamma} \nabla_{\Gamma} X \cdot \nabla_{\Gamma}(X-\mathrm{Id}) \geqslant|X(\Gamma)|-|\Gamma|
$$

The proof of this lemma (see [4, Lemma 1]) relies on the following pointwise estimate.

Lemma 6.2 Let $m=1$ or $m=2, k \in \mathbb{N}$, and $x_{i}, y_{i} \in \mathbb{R}^{k}, i=1, \ldots, m$. Set $(G(x))_{i j}=$ $g_{i j}(x):=x_{i} \cdot x_{j}$ and $g^{i j}=\left(G^{-1}\right)_{i j}$. If $G(y)$ is regular, then

$$
\sqrt{\operatorname{det} G(x)}-\sqrt{\operatorname{det} G(y)} \leqslant \sqrt{\operatorname{det} G(y)} \sum_{l=1}^{k} \sum_{i, j=1}^{m} x_{i}^{l} g^{i j}(y)\left(x_{j}^{l}-y_{j}^{l}\right) .
$$

Proof. See [4, Lemma 1]. The proof is based on purely algebraic arguments: first a suitable vector basis is chosen in order to simplify the inequality, then the statement is shown.

\subsection{The anisotropic case: a new algorithm is needed}

In the anisotropic setting Algorithm 6.1 does not prove to be a stable scheme: numerical experiments show that surfaces tend to "corrugate" quite quickly. Moreover, a rapid degeneration of the grid is often observed.

Inspired by the results described in [8], where the authors analyse a fully discrete numerical scheme approximating the evolution of $n$-dimensional graphs by anisotropic mean curvature, we propose a new scheme. The idea, borrowed from [8], is to add to Algorithm 6.1] a new term weighted by a positive parameter $\sigma$, so that by choosing $\sigma$ large enough (with respect to some values depending on the anisotropy function $F$ ) stability is achieved. Note that the same sort of ideas were used by the author to obtain a stable scheme for the discrete curve shortening flow presented in [15]. Finally, let us remark that for the graphs case described in [8], the authors were able to show both stability and convergence of the fully discrete semi-implicit scheme presented in their work.

The new algorithm we propose is the following.

Algorithm 6.3 Let $M_{h}^{(0)}$ be an initial polyhedron interpolating the initial surface $\mathbf{x}^{(0)}(\tilde{M})$. For a positive parameter $\sigma$ chosen appropriately, compute a sequence of time step solutions $X^{(k)}, k=$ $1,2 \ldots$, such that

$$
\begin{gathered}
\int_{M_{h}^{(k)}} \frac{1}{\tau}\left(X^{(k+1)}-\mathrm{Id}\right) \cdot \Phi \mathrm{d} A+\sigma \int_{M_{h}^{(k)}} F\left(\frac{\partial_{1}^{(k)} \wedge \partial_{2}^{(k)}}{\left|\partial_{1}^{(k)} \wedge \partial_{2}^{(k)}\right|}\right) \nabla_{M_{h}^{(k)}}\left(X^{(k+1)}-\mathrm{Id}\right) \cdot \nabla_{M_{h}^{(k)}} \Phi \mathrm{d} A \\
\quad+\int_{M_{h}^{(k)}} F\left(\frac{\partial_{1}^{(k)} \wedge \partial_{2}^{(k)}}{\left|\partial_{1}^{(k)} \wedge \partial_{2}^{(k)}\right|}\right) \nabla_{M_{h}^{(k)}} \mathrm{Id} \cdot \nabla_{M_{h}^{(k)}} \Phi \mathrm{d} A+\int_{M_{h}^{(k)}} \sum_{i=1}^{n-2} G_{N_{i}^{(k)}}^{(k)}(\Phi) \cdot N_{i}^{(k)} \mathrm{d} A=0
\end{gathered}
$$

for all $\Phi \in\left[\mathcal{V}_{h}\right]^{n}$. The new surface is generated by setting $M_{h}^{(k+1)}=X^{(k+1)}\left(M_{h}^{(k)}\right)$.

Note that for $\sigma=1$ we recover Algorithm 6.1 and that in the isotropic setting stability is achieved for any $\sigma \geqslant 1$. 
Using the mass and stiffness matrices and "right-hand side" defined in 6.5]-6.8, and introducing the new term

$$
U_{l, j}^{(k)}=\int_{M_{h}^{(k)}} F\left(\frac{\partial_{1}^{(k)} \wedge \partial_{2}^{(k)}}{\left|\partial_{1}^{(k)} \wedge \partial_{2}^{(k)}\right|}\right) \nabla_{M_{h}^{(k)}} x_{l} \nabla_{M_{h}^{(k)}} \Phi_{j} \mathrm{~d} A
$$

where $j=1, \ldots, J$ and $l=1, \ldots, n$, we conclude that in each time step we need to solve $n$ linear systems of the form

$$
\left(\frac{1}{\tau} A^{(k)}+\sigma L^{(k)}\right) \bar{X}_{l}^{(k+1)}=\frac{1}{\tau} B_{l}^{(k)}+C_{l}^{(k)}+(\sigma-1) U_{l}^{(k)}
$$

for $l=1, \ldots, n$. Again note that for $\sigma$ positive the matrix $(1 / \tau) A^{(k)}+\sigma L^{(k)}$ is symmetric and positive definite, therefore the above systems admit solutions.

\section{Stability}

Next we prove that Algorithm 6.3 is stable provided $\sigma$ is chosen large enough.

Proposition 7.1 If the positive constant $\sigma$ in Algorithm 6.3 is chosen so that

$$
\begin{aligned}
& \sigma \inf _{\left|p_{1} \wedge p_{2}\right|=1} F\left(p_{1} \wedge p_{2}\right) \\
& \quad \geqslant 22 \max \left\{\sup _{\left|p_{1} \wedge p_{2}\right|=1} F\left(p_{1} \wedge p_{2}\right),\left.\sup _{\left|p_{1} \wedge p_{2}\right|=1}|D F|_{p_{1} \wedge p_{2} \mid}\left|, \sup _{\left|p_{1} \wedge p_{2}\right|=1}\right| D^{2} F\right|_{p_{1} \wedge p_{2} \mid} \mid\right\},
\end{aligned}
$$

then stability is guaranteed, i.e.

$$
\left|M_{h}^{0}\right|_{F} \geqslant \sum_{k=0}^{K} \int_{M_{h}^{(k)}} \frac{1}{\tau}\left|X^{(k+1)}-\mathrm{Id}\right|^{2} \mathrm{~d} A+\left|M_{h}^{K+1}\right|_{F} .
$$

Proof. Test Algorithm 6.3 with $\Phi=X^{(k+1)}-\mathrm{Id}$, use the fact that $G_{N_{i}^{(k)}}^{(k)}(\mathrm{Id}) \cdot N_{i}^{(k)}=0$ for all $i=1, \ldots, n-2$, and obtain

$$
\begin{aligned}
0= & \int_{M_{h}^{(k)}} \frac{1}{\tau}\left|X^{(k+1)}-\mathrm{Id}\right|^{2} \mathrm{~d} A \\
& +\int_{M_{h}^{(k+1)}} F\left(\frac{\partial_{1}^{(k+1)} \wedge \partial_{2}^{(k+1)}}{\left|\partial_{1}^{(k+1)} \wedge \partial_{2}^{(k+1)}\right|}\right) \mathrm{d} A-\int_{M_{h}^{(k)}} F\left(\frac{\partial_{1}^{(k)} \wedge \partial_{2}^{(k)}}{\left|\partial_{1}^{(k)} \wedge \partial_{2}^{(k)}\right|}\right) \mathrm{d} A \\
& +\sigma \int_{M_{h}^{(k)}} F\left(\frac{\partial_{1}^{(k)} \wedge \partial_{2}^{(k)}}{\left|\partial_{1}^{(k)} \wedge \partial_{2}^{(k)}\right|}\right)\left|\nabla_{M_{h}^{(k)}}\left(X^{(k+1)}-\mathrm{Id}\right)\right|^{2} \mathrm{~d} A \\
& +\int_{M_{h}^{(k)}} F\left(\frac{\partial_{1}^{(k)} \wedge \partial_{2}^{(k)}}{\left|\partial_{1}^{(k)} \wedge \partial_{2}^{(k)}\right|}\right) \nabla_{M_{h}^{(k)}} \mathrm{Id} \cdot \nabla_{M_{h}^{(k)}}\left(X^{(k+1)}-\mathrm{Id}\right) \mathrm{d} A \\
& +\int_{M_{h}^{(k)}} \sum_{i=1}^{n-2} G_{N_{i}^{(k)}\left(X^{(k+1)}\right) \cdot N_{i}^{(k)}} \mathrm{d} A \\
& -\int_{M_{h}^{(k+1)}} F\left(\frac{\partial_{1}^{(k+1)} \wedge \partial_{2}^{(k+1)}}{\left|\partial_{1}^{(k+1)} \wedge \partial_{2}^{(k+1)}\right|}\right) \mathrm{d} A+\int_{M_{h}^{(k)}} F\left(\frac{\partial_{1}^{(k)} \wedge \partial_{2}^{(k)}}{\left|\partial_{1}^{(k)} \wedge \partial_{2}^{(k)}\right|}\right) \mathrm{d} A
\end{aligned}
$$


Each integral appearing in the above expression is calculated as a sum of integrals that have a triangle of the discrete surface as parameter domain. A triangle $T_{h}^{(k)} \in M_{h}^{(k)}$ is mapped through $X^{(k+1)}$ onto a triangle $T_{h}^{(k+1)} \in M_{h}^{(k+1)}$. Given a parametrization $\varphi: T \rightarrow T_{h}^{(k)}$ from a reference triangle $T$, the tangential vectors $\partial_{i}^{(k)}$ and $\partial_{i}^{(k+1)}, i=1,2$, are given by $\partial_{i} \varphi$ and $\partial_{i}\left(X^{(k+1)} \circ \varphi\right)$ respectively. Using Proposition 7.5 we can show that the sum of the last five terms is positive provided that $\sigma$ is chosen as in 7.1 . Therefore we get

$$
\begin{aligned}
0 \geqslant & \int_{M_{h}^{(k)}} \frac{1}{\tau}\left|X^{(k+1)}-\mathrm{Id}\right|^{2} \mathrm{~d} A \\
& +\int_{M_{h}^{(k+1)}} F\left(\frac{\partial_{1}^{(k+1)} \wedge \partial_{2}^{(k+1)}}{\left|\partial_{1}^{(k+1)} \wedge \partial_{2}^{(k+1)}\right|}\right) \mathrm{d} A-\int_{M_{h}^{(k)}} F\left(\frac{\partial_{1}^{(k)} \wedge \partial_{2}^{(k)}}{\left|\partial_{1}^{(k)} \wedge \partial_{2}^{(k)}\right|}\right) \mathrm{d} A,
\end{aligned}
$$

and the claim is achieved by summing up over the time steps.

Next we are going to prove Proposition 7.5. We start by giving a few useful lemmas. First of all, we describe a few relations between the norm of the difference of two vectors and the norm of the difference of their normalized counterparts.

Lemma 7.2 Let $v, w \in \mathbb{R}^{n} \backslash\{0\}$ and set $\bar{v}=v /|v|, \bar{w}=w /|w|$. Then

$$
|v-w|^{2}=|v||w||\bar{v}-\bar{w}|^{2}+(|v|-|w|)^{2} .
$$

In particular, for $v$ a unit vector we have

$$
\begin{aligned}
& |v-w|^{2} \geqslant|w||v-\bar{w}|^{2}, \\
& 2|v-w| \geqslant|v-\bar{w}| .
\end{aligned}
$$

Proof. The proof is elementary and left to the reader.

The next lemma is nothing deep, but it makes reading the proof of Proposition 7.5 somewhat easier.

LEMma 7.3 Let $\left\{x_{i}, y_{i} \in \mathbb{R}^{n} \backslash\{0\}, i=1,2\right\}$ be a set of linearly independent vectors and let $F$ be an anisotropy function (as in Definition 2.2). Set

$$
\begin{array}{lll}
A(s):=y_{1} \wedge\left((1-s) \frac{x_{2}}{\left|x_{2}\right|}+s y_{2}\right), & B(s):=\left((1-s) \frac{x_{1}}{\left|x_{1}\right|}+s y_{1}\right) \wedge y_{2}, \\
C(s):=x_{1} \wedge\left(s x_{2}+(1-s) y_{2}\right), & D(s):=y_{1} \wedge\left(s x_{2}+(1-s) y_{2}\right), \\
E(s):=\left(s x_{1}+(1-s) y_{1}\right) \wedge x_{2}, & G(s):=\left(s x_{1}+(1-s) y_{1}\right) \wedge y_{2},
\end{array}
$$

where $s \in[0,1]$. Then

$$
\begin{aligned}
& F\left(y_{1} \wedge x_{2}\right)=\left.D F\right|_{y_{1} \wedge y_{2}}\left(y_{1} \wedge x_{2}\right)+\left.\frac{\left|x_{2}\right|}{2} D^{2} F\right|_{A}\left(y_{1} \wedge\left(\frac{x_{2}}{\left|x_{2}\right|}-y_{2}\right)\right)^{2}, \\
& F\left(x_{1} \wedge y_{2}\right)=\left.D F\right|_{y_{1} \wedge y_{2}}\left(x_{1} \wedge y_{2}\right)+\left.\frac{\left|x_{1}\right|}{2} D^{2} F\right|_{B}\left(\left(\frac{x_{1}}{\left|x_{1}\right|}-y_{1}\right) \wedge y_{2}\right)^{2}, \\
& F\left(x_{1} \wedge y_{2}\right)-F\left(x_{1} \wedge x_{2}\right)=\left.D F\right|_{x_{1} \wedge x_{2}}\left(x_{1} \wedge\left(y_{2}-x_{2}\right)\right)+\left.\frac{1}{2} D^{2} F\right|_{C}\left(x_{1} \wedge\left(y_{2}-x_{2}\right)\right)^{2},
\end{aligned}
$$




$$
\begin{aligned}
& F\left(y_{1} \wedge x_{2}\right)-F\left(y_{1} \wedge y_{2}\right)=\left.D F\right|_{y_{1} \wedge y_{2}}\left(y_{1} \wedge\left(x_{2}-y_{2}\right)\right)+\left.\frac{1}{2} D^{2} F\right|_{D}\left(y_{1} \wedge\left(x_{2}-y_{2}\right)\right)^{2}, \\
& F\left(y_{1} \wedge x_{2}\right)-F\left(x_{1} \wedge x_{2}\right)=\left.D F\right|_{x_{1} \wedge x_{2}}\left(\left(y_{1}-x_{1}\right) \wedge x_{2}\right)+\left.\frac{1}{2} D^{2} F\right|_{E}\left(\left(y_{1}-x_{1}\right) \wedge x_{2}\right)^{2}, \\
& F\left(x_{1} \wedge y_{2}\right)-F\left(y_{1} \wedge y_{2}\right)=\left.D F\right|_{y_{1} \wedge y_{2}}\left(\left(x_{1}-y_{1}\right) \wedge y_{2}\right)+\left.\frac{1}{2} D^{2} F\right|_{G}\left(\left(x_{1}-y_{1}\right) \wedge y_{2}\right)^{2},
\end{aligned}
$$

where $A=A\left(s_{a}\right), B=B\left(s_{b}\right), C=C\left(s_{c}\right), D=D\left(s_{d}\right), E=E\left(s_{e}\right), G=G\left(s_{g}\right)$ for some $s_{a}, s_{b}, s_{c}, s_{d}, s_{e}, s_{g} \in[0,1]$.

Proof. The claims are obtained by using a Taylor expansion and the homogeneity properties of $F$. For example, for (7.5) one computes the Taylor expansion

$$
\begin{aligned}
F\left(y_{1} \wedge \frac{x_{2}}{\left|x_{2}\right|}\right)= & F\left(y_{1} \wedge y_{2}\right)+\left.D F\right|_{y_{1} \wedge y_{2}}\left(y_{1} \wedge\left(\frac{x_{2}}{\left|x_{2}\right|}-y_{2}\right)\right) \\
& +\left.\frac{1}{2} D^{2} F\right|_{A}\left(y_{1} \wedge\left(\frac{x_{2}}{\left|x_{2}\right|}-y_{2}\right)\right)\left(y_{1} \wedge\left(\frac{x_{2}}{\left|x_{2}\right|}-y_{2}\right)\right),
\end{aligned}
$$

where $A$ is some point on the segment $\left[y_{1} \wedge x_{2} /\left|x_{2}\right|, y_{1} \wedge y_{2}\right]$. Using 2.3 we get

$$
F\left(y_{1} \wedge \frac{x_{2}}{\left|x_{2}\right|}\right)=\left.D F\right|_{y_{1} \wedge y_{2}}\left(y_{1} \wedge \frac{x_{2}}{\left|x_{2}\right|}\right)+\left.\frac{1}{2} D^{2} F\right|_{A}\left(y_{1} \wedge\left(\frac{x_{2}}{\left|x_{2}\right|}-y_{2}\right)\right)^{2},
$$

and $(7.5)$ is obtained after multiplying the above by $\left|x_{2}\right|$ and using the homogeneity of $F$. Note that in view of Theorem 3.1 and the fact that the vectors $x_{1}, x_{2}, y_{1}, y_{2}$ are linearly independent we do not differentiate at the origin.

We will need to estimate the norm $|v \wedge w|$ from below.

LEMmA 7.4 If $v, w \in \mathbb{R}^{n} \backslash\{0\}$ are such that $\left|\frac{v}{|v|} \cdot \frac{w}{|w|}\right| \leqslant \delta$ for some $0 \leqslant \delta \leqslant 1$, then

$$
|v \wedge w| \geqslant|v||w| \sqrt{1-\delta^{2}}
$$

Moreover, $\left|\frac{v}{|v|} \cdot \frac{w}{|w|}\right| \leqslant \delta$ if and only if $\sqrt{2} \sqrt{1-\delta} \leqslant\left|\frac{v}{|v|}-\frac{w}{|w|}\right| \leqslant \sqrt{2} \sqrt{1+\delta}$.

Proof. Setting $\bar{v}=v /|v|$, we just observe that

$$
|v \wedge w|=|v||w| \sqrt{1-(\bar{v} \cdot \bar{w})^{2}}
$$

The final remark follows from a simple geometrical argument. Indeed, if we let $l=\bar{v} \cdot \bar{w}$ be the (signed) projection of $\bar{w}$ onto $\bar{v}$, then we immediately derive that $|\bar{v}-\bar{w}|=\sqrt{(1-l)^{2}+\left(1-l^{2}\right)}=$ $\sqrt{2} \sqrt{1-l}$ and the claim follows.

Proposition 7.5 Let $x_{i}, y_{i} \in \mathbb{R}^{n} \backslash\{0\}, i=1,2$, and set $(G(x))_{i j}=x_{i} \cdot x_{j}$ and $g^{i j}=\left(G^{-1}\right)_{i j}$. Assume that the matrices $G(y)$ and $G(x)$ are regular and let $\left\{v_{1}, \ldots, v_{n-2}\right\}$ be a set of orthonormal 
vectors such that $v_{i} \cdot y_{1}=0=v_{i} \cdot y_{2}$ for every $i=1, \ldots, n-2$. Let $F: \Lambda^{2} \mathbb{R}^{n} \rightarrow[0, \infty)$ be an anisotropy function. Then there exists $\sigma>0$ such that

$$
\begin{aligned}
\left|y_{1} \wedge y_{2}\right|\{\sigma & F\left(\frac{y_{1} \wedge y_{2}}{\left|y_{1} \wedge y_{2}\right|}\right) \sum_{i, j=1}^{2} g^{i j}(y)\left(x_{i}-y_{i}\right) \cdot\left(x_{j}-y_{j}\right)+F\left(\frac{y_{1} \wedge y_{2}}{\left|y_{1} \wedge y_{2}\right|}\right) \\
& +F\left(\frac{y_{1} \wedge y_{2}}{\left|y_{1} \wedge y_{2}\right|}\right) \sum_{i, j=1}^{2} g^{i j}(y)\left(x_{j}-y_{j}\right) \cdot y_{i} \\
& \left.+\sum_{i=1}^{n-2} \frac{1}{\left|y_{1} \wedge y_{2}\right|}\left(\left.D F\right|_{y_{1} \wedge y_{2}}\left(v_{i} \wedge y_{2}\right) x_{1} \cdot v_{i}+\left.D F\right|_{y_{1} \wedge y_{2}}\left(y_{1} \wedge v_{i}\right) x_{2} \cdot v_{i}\right)\right\} \\
& -F\left(\frac{x_{1} \wedge x_{2}}{\left|x_{1} \wedge x_{2}\right|}\right)\left|x_{1} \wedge x_{2}\right| \geqslant 0 .
\end{aligned}
$$

More precisely, the above inequality is satisfied for $\sigma$ such that

$$
\begin{aligned}
\sigma \inf _{\left|p_{1} \wedge p_{2}\right|=1} F\left(p_{1} \wedge p_{2}\right) \\
\quad \geqslant 22 \max \left\{\sup _{\left|p_{1} \wedge p_{2}\right|=1} F\left(p_{1} \wedge p_{2}\right),\left.\sup _{\left|p_{1} \wedge p_{2}\right|=1}|D F|_{p_{1} \wedge p_{2} \mid}\left|, \sup _{\left|p_{1} \wedge p_{2}\right|=1}\right| D^{2} F\right|_{p_{1} \wedge p_{2} \mid} \mid\right\} .
\end{aligned}
$$

Proof. Step 1: We can assume that $(G(y))_{i j}=\delta_{i j}$. Indeed, $G(y)$ is a symmetric real matrix and as such it can be diagonalized. Let $A:=\left(\begin{array}{ll}a & b \\ c & d\end{array}\right)$ be an orthogonal matrix such that $D:=A^{T} G(y) A$ with $D$ diagonal, $D=\operatorname{diag}\left(d_{1}, d_{2}\right)$. Possibly after changing the sign of a column of $A$, we can assume that $\operatorname{det}(A)=1$. After setting

$$
\begin{array}{ll}
y_{1}=a \tilde{y}_{1}+b \tilde{y}_{2}, & x_{1}=a \tilde{x}_{1}+b \tilde{x}_{2}, \\
y_{2}=c \tilde{y}_{1}+d \tilde{y}_{2}, & x_{2}=c \tilde{x}_{1}+d \tilde{x}_{2},
\end{array}
$$

a quick calculation gives $\tilde{y}_{i} \cdot \tilde{y}_{j}=\left|\tilde{y}_{i}{ }^{2}\right| \delta_{i j}, d_{i}=\left|\tilde{y}_{i}\right|^{2}$, and $(G(y))^{-1}=A D^{-1} A^{T}$. Recall also that $y_{1} \wedge y_{2}=\operatorname{det}(A) \tilde{y}_{1} \wedge \tilde{y}_{2}$ and $\left|y_{1} \wedge y_{2}\right|=\sqrt{\operatorname{det}(G(y))}$. Using the fact that $\operatorname{det}(A)>0$ and the homogeneity properties of $F$, we can easily transform inequality (7.12) into

$$
\begin{aligned}
&\left|\tilde{y}_{1}\right|\left|\tilde{y}_{2}\right|\left(\sigma F\left(\frac{\tilde{y}_{1} \wedge \tilde{y}_{2}}{\left|\tilde{y}_{1} \wedge \tilde{y}_{2}\right|}\right) \sum_{i=1}^{2}\left|\tilde{y}_{i}\right|^{-2}\left|\tilde{x}_{i}-\tilde{y}_{i}\right|^{2}+F\left(\frac{\tilde{y}_{1} \wedge \tilde{y}_{2}}{\left|\tilde{y}_{1} \wedge \tilde{y}_{2}\right|}\right)\right. \\
&+F\left(\frac{\tilde{y}_{1} \wedge \tilde{y}_{2}}{\left|\tilde{y}_{1} \wedge \tilde{y}_{2}\right|}\right) \sum_{i=1}^{2}\left|\tilde{y}_{i}\right|^{-2}\left(\tilde{x}_{i}-\tilde{y}_{i}\right) \cdot \tilde{y}_{i} \\
&\left.+\sum_{i=1}^{n-2} \frac{1}{\left|\tilde{y}_{1}\right|\left|\tilde{y}_{2}\right|}\left(D F\left|\tilde{y}_{1} \wedge \tilde{y}_{2}\left(v_{i} \wedge \tilde{y}_{2}\right) \tilde{x}_{1} \cdot v_{i}+D F\right|_{\tilde{y}_{1} \wedge \tilde{y}_{2}}\left(\tilde{y}_{1} \wedge v_{i}\right) \tilde{x}_{2} \cdot v_{i}\right)\right) \\
&- F\left(\frac{\tilde{x}_{1} \wedge \tilde{x}_{2}}{\left|\tilde{x}_{1} \wedge \tilde{x}_{2}\right|}\right)\left|\tilde{x}_{1} \wedge \tilde{x}_{2}\right| \geqslant 0 .
\end{aligned}
$$

Eventually by setting $\hat{x}_{i}:=\tilde{x}_{i} /\left|\tilde{y}_{i}\right|$ and $\hat{y}_{i}:=\tilde{y}_{i} /\left|\tilde{y}_{i}\right|$ we obtain the desired form. 
Step 2: Let us go back to inequality $(7.12)$ and assume that $G(y)$ is the identity matrix. We need to show that there exists a positive $\sigma$ such that

$$
\begin{aligned}
\sigma F\left(y_{1} \wedge y_{2}\right) \sum_{i=1}^{2}\left|x_{i}-y_{i}\right|^{2}+F( & \left.y_{1} \wedge y_{2}\right) \sum_{i=1}^{2}\left(x_{i}-y_{i}\right) \cdot y_{i} \\
& +\sum_{i=1}^{n-2}\left(\left.D F\right|_{y_{1} \wedge y_{2}}\left(v_{i} \wedge y_{2}\right) x_{1} \cdot v_{i}+\left.D F\right|_{y_{1} \wedge y_{2}}\left(y_{1} \wedge v_{i}\right) x_{2} \cdot v_{i}\right) \\
& +F\left(y_{1} \wedge y_{2}\right)-F\left(\frac{x_{1} \wedge x_{2}}{\left|x_{1} \wedge x_{2}\right|}\right)\left|x_{1} \wedge x_{2}\right| \geqslant 0 .
\end{aligned}
$$

Note that $\left\{y_{1}, y_{2}, v_{1}, \ldots, v_{n-2}\right\}$ is an orthonormal basis for $\mathbb{R}^{n}$ and $\left\{y_{1} \wedge y_{2}, y_{1} \wedge v_{1}, \ldots, y_{1} \wedge v_{n-2}\right.$, $\left.v_{1} \wedge y_{2}, \ldots, v_{n-2} \wedge y_{2}, v_{i} \wedge v_{j}\right\}$ with $1 \leqslant i<j \leqslant n-2$ is an orthonormal basis for $\Lambda^{2} \mathbb{R}^{n}$. Using the fact that

$$
\begin{aligned}
\left.D F\right|_{y_{1} \wedge y_{2}}(\cdot) & =\left\langle\nabla F\left(y_{1} \wedge y_{2}\right), \cdot\right\rangle \\
& =\left\langle\left\langle\nabla F\left(y_{1} \wedge y_{2}\right), y_{1} \wedge y_{2}\right\rangle y_{1} \wedge y_{2}+\left\langle\nabla F\left(y_{1} \wedge y_{2}\right), y_{1} \wedge v_{1}\right\rangle y_{1} \wedge v_{1}+\cdots, \cdot\right\rangle
\end{aligned}
$$

one quickly verifies that

$$
\begin{aligned}
\left.D F\right|_{y_{1} \wedge y_{2}}\left(y_{1} \wedge x_{2}+x_{1} \wedge y_{2}\right)=F( & \left.y_{1} \wedge y_{2}\right)\left(x_{1} \cdot y_{1}+x_{2} \cdot y_{2}\right) \\
& +\sum_{i=1}^{n-2}\left(\left.D F\right|_{y_{1} \wedge y_{2}}\left(v_{i} \wedge y_{2}\right) x_{1} \cdot v_{i}+\left.D F\right|_{y_{1} \wedge y_{2}}\left(y_{1} \wedge v_{i}\right) x_{2} \cdot v_{i}\right),
\end{aligned}
$$

so that the problem now boils down to finding a $\sigma>0$ such that

$$
\begin{aligned}
\sigma F\left(y_{1} \wedge y_{2}\right) \sum_{i=1}^{2}\left|x_{i}-y_{i}\right|^{2}+\left.D F\right|_{y_{1} \wedge y_{2}}\left(y_{1} \wedge x_{2}+x_{1} \wedge y_{2}\right) & \\
& -F\left(y_{1} \wedge y_{2}\right)-F\left(\frac{x_{1} \wedge x_{2}}{\left|x_{1} \wedge x_{2}\right|}\right)\left|x_{1} \wedge x_{2}\right| \geqslant 0
\end{aligned}
$$

Step 3: We now distinguish between different cases. As before, we set $\bar{v}:=v /|v|$ for $v \in \mathbb{R}^{n} \backslash\{0\}$.

Case (a): Assume that $\left|\bar{x}_{i}-y_{i}\right|^{2}<\alpha, i=1,2$, with $0<\alpha<4$ to be chosen later. Note that for $s \in[0,1]$ we have

$$
\left|(1-s) y_{i}+s \bar{x}_{i}\right|^{2}=1-s(1-s)\left|\bar{x}_{i}-y_{i}\right|^{2} \geqslant 1-s(1-s) \alpha \geqslant 1-\alpha / 4 .
$$

In the following we will need to give a positive bound from below for $|A(s)|$ and $|B(s)|$, where $A(s)$ and $B(s)$ are defined as in Lemma 7.3 and $s \in[0,1]$. With $\left|y_{1} \cdot \bar{x}_{2}\right| \leqslant \delta$ for some $0 \leqslant \delta \leqslant 1$, we can infer that

$$
\begin{aligned}
|A(s)|^{2} & =\left|(1-s) \bar{x}_{2}+s y_{2}\right|^{2}-(1-s)^{2}\left(y_{1} \cdot \bar{x}_{2}\right)^{2} \\
& =1-s(1-s)\left|\bar{x}_{2}-y_{2}\right|^{2}-(1-s)^{2}\left(y_{1} \cdot \bar{x}_{2}\right)^{2} \\
& \geqslant 1-s(1-s) \alpha-(1-s)^{2} \delta^{2}=s^{2}\left(\alpha-\delta^{2}\right)+s\left(2 \delta^{2}-\alpha\right)+1-\delta^{2} .
\end{aligned}
$$

Looking at the case where $s=0$, we deduce that we certainly need $0 \leqslant \delta<1$. 
We next show that by choosing $\alpha$ small enough we can control $\delta$ and ensure that $|A(s)|,|B(s)| \geqslant$ $c_{\alpha}>0$.

Motivated by the fact that $\left|y_{1} \cdot \bar{x}_{2}\right| \leqslant \delta$ is equivalent to requiring that $\sqrt{2(1-\delta)} \leqslant\left|y_{1}-\bar{x}_{2}\right| \leqslant$ $\sqrt{2(1+\delta)}$ (see Lemma 7.4), we give the following definitions.

Let $B_{y_{i}}(\sqrt{\alpha})$ be the closed ball

$$
B_{y_{i}}(\sqrt{\alpha})=\left\{\xi \in \mathbb{R}^{n}:\left|\xi-y_{i}\right|^{2} \leqslant \alpha\right\}
$$

for $i=1,2$. By assumption $y_{i}, \bar{x}_{i} \in B_{y_{i}}(\sqrt{\alpha}) \cap S^{n-1}$. Let $Q_{i}$ be the "cap"

$$
Q_{i}:=S^{n-1} \cap B_{y_{i}}(\sqrt{\alpha}) \text {. }
$$

The sets $Q_{i}, i=1,2$, are compact and not empty. Finally, let $C_{i}^{+}$be the cone

$$
C_{i}^{+}:=\left\{\xi \in \mathbb{R}^{n} \backslash\{0\}: \xi /|\xi| \in Q_{i}\right\} \cup\{0\},
$$

and $C_{i}$ be the double cone

$$
C_{i}:=C_{i}^{+} \cup\left\{\xi \in \mathbb{R}^{n}:-\xi \in C_{i}^{+}\right\}
$$

for $i=1,2$.

First we observe that, by assuming that $\alpha<2$, we can avoid the cases where $y_{1}$ might coincide with $\pm \bar{x}_{2}$ (or $y_{2}= \pm \bar{x}_{1}$ ) and $\delta$ be equal to one.

Next let $l \in(0,1)$ be such that $\alpha=2(1-l)$. By using a coordinate system such that $y_{1}=$ $(1,0,0, \ldots, 0)$ and $y_{2}=(0,1,0, \ldots, 0)$ we can easily infer that

$$
\begin{aligned}
& Q_{1}=\left\{\xi=\left(\xi_{1}, \ldots, \xi_{n}\right) \in S^{n-1}: l \leqslant \xi_{1} \leqslant 1\right\}, \\
& Q_{2}=\left\{\xi=\left(\xi_{1}, \ldots, \xi_{n}\right) \in S^{n-1}: l \leqslant \xi_{2} \leqslant 1\right\} .
\end{aligned}
$$

Moreover, note that since $\bar{x}_{1} \in Q_{1}$ we have

$$
\max _{\xi \in Q_{1}}\left|y_{2}-\xi\right| \geqslant\left|y_{2}-\bar{x}_{1}\right| \geqslant \min _{\xi \in Q_{1}}\left|y_{2}-\xi\right| \text {. }
$$

Now for $\xi \in Q_{1}$ we have $\left|y_{2}-\xi\right|=\sqrt{\xi_{1}^{2}+\left(1-\xi_{2}\right)^{2}+\ldots+\xi_{n}^{2}}=\sqrt{2-2 \xi_{2}}$, and since $l^{2} \leqslant$ $\xi_{1}^{2} \leqslant 1$ we infer $1-l^{2} \geqslant 1-\xi_{1}^{2}-\xi_{3}^{2}-\cdots-\xi_{n}^{2}=\xi_{2}^{2} \geqslant 0$, that is, $\sqrt{1-l^{2}} \geqslant\left|\xi_{2}\right|$. This implies that

$$
\sqrt{2+2 \sqrt{1-l^{2}}} \geqslant \sqrt{2+2\left|\xi_{2}\right|} \geqslant\left|y_{2}-\xi\right|=\sqrt{2-2 \xi_{2}} \geqslant \sqrt{2-2\left|\xi_{2}\right|} \geqslant \sqrt{2-2 \sqrt{1-l^{2}}}
$$

Using similar arguments for $y_{1}$ and $\bar{x}_{2}$ we finally obtain

$$
\begin{aligned}
& \sqrt{2+2 \sqrt{1-l^{2}}} \geqslant\left|y_{2}-\bar{x}_{1}\right| \geqslant \sqrt{2-2 \sqrt{1-l^{2}}}, \\
& \sqrt{2+2 \sqrt{1-l^{2}}} \geqslant\left|y_{1}-\bar{x}_{2}\right| \geqslant \sqrt{2-2 \sqrt{1-l^{2}}},
\end{aligned}
$$

which (again by Lemma 7.4) is equivalent to $\left|y_{1} \cdot \bar{x}_{2}\right|,\left|y_{2} \cdot \bar{x}_{1}\right| \leqslant \sqrt{1-l^{2}}=\sqrt{\alpha(1-\alpha / 4)}$, since $l=1-\alpha / 2$. With $\delta=\delta(\alpha)=\sqrt{\alpha(1-\alpha / 4)}$ and $0<\alpha<2$, observe that $|A(s)|^{2}=s^{2}\left(\alpha-\delta^{2}\right)+$ $s\left(2 \delta^{2}-\alpha\right)+1-\delta^{2} \geqslant 1-\delta^{2}=(1-\alpha / 2)^{2}$ for all $s \in[0,1]$. 
Summing up, we have shown that for $0<\alpha<2,|A(s)| \geqslant c_{\alpha}$ with $c_{\alpha}=\sqrt{1-\delta^{2}}=1-\alpha / 2$. Similar arguments lead to the estimate $|B(s)| \geqslant c_{\alpha}$.

Finally, let us remark that by making $\alpha$ even smaller we can ensure that $C(s), D(s), E(s)$, $G(s)$ (as defined in Lemma 7.3 do not vanish for any choice of $s \in[0,1]$. For example, consider $C(s)=x_{1} \wedge\left(s x_{2}+(1-s) y_{2}\right)$ for a fixed $s \in[0,1]$. By assumption $x_{1} \in Q_{1} \subset C_{1} \backslash\{0\}$. By Theorem 3.1. $C(s)=0$ if and only if there exists $b \neq 0$ such that $b x_{1}=s x_{2}+(1-s) y_{2}$, i.e. the vectors are linearly dependent. However, since $s x_{2}+(1-s) y_{2} \in C_{2}^{+}$, the linear dependence can be expressed by saying that $x_{1}$ also lies in the cone $C_{2}$. Therefore $x_{1} \in C_{1} \backslash\{0\} \cap C_{2}$. By taking $\alpha$ so small that $Q_{1} \cap Q_{2}=\emptyset$ we can ensure that $C_{1} \cap C_{2}=\{0\}$ and thus $C(s) \neq 0$. It is easy to verify that $Q_{1} \cap Q_{2}=\emptyset$ provided that $\alpha<2-\sqrt{2}$.

Under this assumption and by applying similar arguments we also deduce that $D(s), E(s), G(s) \neq 0$ for any $s \in[0,1]$. These facts will be used in the following computations.

Thus let $0<\alpha<2-\sqrt{2}$, with $\alpha$ to be chosen later. In the following we write $\inf _{S^{1}} F$ and $\sup _{S^{1}} F$ for $\inf _{\left|p_{1} \wedge p_{2}\right|=1} F\left(p_{1} \wedge p_{2}\right)$ and $\sup _{\left|p_{1} \wedge p_{2}\right|=1} F\left(p_{1} \wedge p_{2}\right)$; $\sup _{S^{1}}|D F|$ for $\sup _{\left|p_{1} \wedge p_{2}\right|=1}|D F|_{p_{1} \wedge p_{2}} \mid$; and $\sup _{S^{1}}\left|D^{2} F\right|$ for $\sup _{\left|p_{1} \wedge p_{2}\right|=1}\left|D^{2} F\right|_{p_{1} \wedge p_{2} \mid} \mid$. We have

$$
\begin{aligned}
& \sigma F\left(y_{1} \wedge y_{2}\right) \sum_{i=1}^{2}\left|x_{i}-y_{i}\right|^{2}+\left.D F\right|_{y_{1} \wedge y_{2}}\left(y_{1} \wedge x_{2}\right)+\left.D F\right|_{y_{1} \wedge y_{2}}\left(x_{1} \wedge y_{2}\right) \\
& =\sigma F\left(y_{1} \wedge y_{2}\right) \sum_{i=1}^{2}\left|x_{i}-y_{i}\right|^{2}+F\left(y_{1} \wedge x_{2}\right)-\left.\frac{\left|x_{2}\right|}{2} D^{2} F\right|_{A}\left(y_{1} \wedge\left(\bar{x}_{2}-y_{2}\right)\right)^{2} \\
& \quad+F\left(x_{1} \wedge y_{2}\right)-\left.\frac{\left|x_{1}\right|}{2} D^{2} F\right|_{B}\left(\left(\bar{x}_{1}-y_{1}\right) \wedge y_{2}\right)^{2} \\
& \quad-F\left(y_{1} \wedge y_{2}\right)-F\left(x_{1} \wedge x_{2}\right)
\end{aligned}
$$$$
=\sigma F\left(y_{1} \wedge y_{2}\right) \sum_{i=1}^{2}\left|x_{i}-y_{i}\right|^{2}+\left.D F\right|_{x_{1} \wedge x_{2}}\left(x_{1} \wedge\left(y_{2}-x_{2}\right)\right)+\left.\frac{1}{2} D^{2} F\right|_{C}\left(x_{1} \wedge\left(y_{2}-x_{2}\right)\right)^{2}
$$$$
+\left.D F\right|_{y_{1} \wedge y_{2}}\left(y_{1} \wedge\left(x_{2}-y_{2}\right)\right)+\left.\frac{1}{2} D^{2} F\right|_{D}\left(y_{1} \wedge\left(x_{2}-y_{2}\right)\right)^{2}
$$$$
-\left.\frac{\left|x_{2}\right|}{2} D^{2} F\right|_{A}\left(y_{1} \wedge\left(\bar{x}_{2}-y_{2}\right)\right)^{2}-\left.\frac{\left|x_{1}\right|}{2} D^{2} F\right|_{B}\left(\left(\bar{x}_{1}-y_{1}\right) \wedge y_{2}\right)^{2}
$$

$\geqslant \sigma F\left(y_{1} \wedge y_{2}\right) \sum_{i=1}^{2}\left|x_{i}-y_{i}\right|^{2}-\left.\frac{\left|x_{2}\right|}{2|A|} D^{2} F\right|_{A /|A|}\left(y_{1} \wedge\left(\bar{x}_{2}-y_{2}\right)\right)^{2}-\left.\frac{\left|x_{1}\right|}{2|B|} D^{2} F\right|_{B /|B|}\left(\left(\bar{x}_{1}-y_{1}\right) \wedge y_{2}\right)^{2}$

$+\left(\left.D F\right|_{y_{1} \wedge y_{2}}-\left.D F\right|_{y_{1} \wedge \bar{x}_{2}}\right)\left(y_{1} \wedge\left(x_{2}-y_{2}\right)\right)+\left(\left.D F\right|_{y_{1} \wedge \bar{x}_{2}}-\left.D F\right|_{\bar{x}_{1} \wedge \bar{x}_{2}}\right)\left(y_{1} \wedge\left(x_{2}-y_{2}\right)\right)$

$-D F_{\bar{x}_{1} \wedge \bar{x}_{2}}\left(\left(x_{1}-y_{1}\right) \wedge\left(x_{2}-y_{2}\right)\right)$

(by the convexity of $F$ and (2.4))

$\geqslant \sigma \inf _{S^{1}} F \sum_{i=1}^{2}\left|x_{i}-y_{i}\right|^{2}-\sup _{S^{1}}\left|D^{2} F\right|\left(\frac{\left|x_{2}\right|}{2|A|}\left|\bar{x}_{2}-y_{2}\right|^{2}+\frac{\left|x_{1}\right|}{2|B|}\left|\bar{x}_{1}-y_{1}\right|^{2}\right)$

$-\sup _{S^{1}}|D F|\left(\frac{1}{2}\left|x_{1}-y_{1}\right|^{2}+\frac{1}{2}\left|x_{2}-y_{2}\right|^{2}\right)$

$-\int_{0}^{1} D^{2} F\left(\frac{A(s)}{|A(s)|}\right) \frac{1}{|A(s)|} \mathrm{d} s\left(y_{1} \wedge\left(\bar{x}_{2}-y_{2}\right)\right)\left(y_{1} \wedge\left(x_{2}-y_{2}\right)\right)$

$-\int_{0}^{1} D^{2} F\left(\frac{B(s)}{|B(s)|}\right) \frac{1}{|B(s)|} \mathrm{d} s\left(\left(\bar{x}_{1}-y_{1}\right) \wedge \bar{x}_{2}\right)\left(y_{1} \wedge\left(x_{2}-y_{2}\right)\right)$

(by (2.4)) 


$$
\begin{aligned}
& \geqslant\left(\sigma \inf _{S^{1}} F-\sup _{S^{1}}\left|D^{2} F\right| \frac{1}{2 c_{\alpha}}-\frac{1}{2} \sup _{S^{1}}|D F|\right) \sum_{i=1}^{2}\left|x_{i}-y_{i}\right|^{2}-\sup _{S^{1}}\left|D^{2} F\right| \frac{1}{c_{\alpha}}\left|\bar{x}_{2}-y_{2}\right|\left|x_{2}-y_{2}\right| \\
& \left.-\sup _{S^{1}}\left|D^{2} F\right| \frac{1}{c_{\alpha}}\left|\bar{x}_{1}-y_{1}\right|\left|x_{2}-y_{2}\right| \quad \text { (by } 7.3 \text { and since }|A(s)|,|B(s)| \geqslant c_{\alpha}\right) \\
& \geqslant\left(\sigma \inf _{S^{1}} F-\sup _{S^{1}}\left|D^{2} F\right| \frac{3}{2 c_{\alpha}}-\frac{1}{2} \sup _{S^{1}}|D F|\right) \sum_{i=1}^{2}\left|x_{i}-y_{i}\right|^{2}-\sup _{S^{1}}\left|D^{2} F\right| \frac{2}{c_{\alpha}}\left|x_{2}-y_{2}\right|^{2}
\end{aligned}
$$

by (7.4) and Young's inequality. Thus we have shown that

$$
\begin{gathered}
\sigma F\left(y_{1} \wedge y_{2}\right) \sum_{i=1}^{2}\left|x_{i}-y_{i}\right|^{2}+\left.D F\right|_{y_{1} \wedge y_{2}}\left(y_{1} \wedge x_{2}\right)+\left.D F\right|_{y_{1} \wedge y_{2}}\left(x_{1} \wedge y_{2}\right)-F\left(y_{1} \wedge y_{2}\right)-F\left(x_{1} \wedge x_{2}\right) \\
\geqslant\left(\sigma \inf _{S^{1}} F-\sup _{S^{1}}\left|D^{2} F\right| \frac{3}{2 c_{\alpha}}-\frac{1}{2} \sup _{S^{1}}|D F|\right) \sum_{i=1}^{2}\left|x_{i}-y_{i}\right|^{2}-\sup _{S^{1}}\left|D^{2} F\right| \frac{2}{c_{\alpha}}\left|x_{2}-y_{2}\right|^{2} .
\end{gathered}
$$

On the other hand, one could use (7.9) and (7.10) instead of (7.7) and (7.8) and obtain

$$
\begin{gathered}
\sigma F\left(y_{1} \wedge y_{2}\right) \sum_{i=1}^{2}\left|x_{i}-y_{i}\right|^{2}+\left.D F\right|_{y_{1} \wedge y_{2}}\left(y_{1} \wedge x_{2}\right)+\left.D F\right|_{y_{1} \wedge y_{2}}\left(x_{1} \wedge y_{2}\right) \\
-F\left(y_{1} \wedge y_{2}\right)-F\left(x_{1} \wedge x_{2}\right) \\
\geqslant\left(\sigma \inf _{S^{1}} F-\sup _{S^{1}}\left|D^{2} F\right| \frac{3}{2 c_{\alpha}}-\frac{1}{2} \sup _{S^{1}}|D F|\right) \sum_{i=1}^{2}\left|x_{i}-y_{i}\right|^{2}-\sup _{S^{1}}\left|D^{2} F\right| \frac{2}{c_{\alpha}}\left|x_{1}-y_{1}\right|^{2},
\end{gathered}
$$

so that, after adding $(7.16)$ and $(7.17)$, and recalling that $c_{\alpha}=1-\alpha / 2$, we can finally state that for some $0<\alpha<2-\sqrt{2}$ (to be chosen later) we have

$$
\begin{aligned}
\sigma F\left(y_{1} \wedge y_{2}\right) \sum_{i=1}^{2}\left|x_{i}-y_{i}\right|^{2} & +\left.D F\right|_{y_{1} \wedge y_{2}}\left(y_{1} \wedge x_{2}\right)+\left.D F\right|_{y_{1} \wedge y_{2}}\left(x_{1} \wedge y_{2}\right)-F\left(y_{1} \wedge y_{2}\right)-F\left(x_{1} \wedge x_{2}\right) \\
& \geqslant\left(\sigma \inf _{S^{1}} F-\sup _{S^{1}}\left|D^{2} F\right| \frac{5}{2 c_{\alpha}}-\frac{1}{2} \sup _{S^{1}}|D F|\right) \sum_{i=1}^{2}\left|x_{i}-y_{i}\right|^{2} \\
& \geqslant\left(\sigma \inf _{S^{1}} F-\max \left\{\sup _{S^{1}}\left|D^{2} F\right|, \sup _{S^{1}}|D F|\right\}\left(\frac{1}{2}+\frac{5}{2-\alpha}\right)\right) \sum_{i=1}^{2}\left|x_{i}-y_{i}\right|^{2},
\end{aligned}
$$

which is positive provided $\sigma$ is chosen large enough.

Case (b): Assume now that $\left|\bar{x}_{i}-y_{i}\right|^{2} \geqslant \alpha, i=1,2$, with $4>\alpha>0$ to be chosen later. Then in particular we have $1 / \alpha \geqslant 1 /\left|\bar{x}_{i}-y_{i}\right|^{2}$. 
Note also that by using (7.4) we obtain

$$
\begin{aligned}
\left|x_{1} \wedge x_{2}\right| \leqslant & \left|x_{1}\right|\left|x_{2}\right| \leqslant\left|x_{1}-y_{1}\right|\left|x_{2}-y_{2}\right|+\left|x_{1}-y_{1}\right|+\left|x_{2}-y_{2}\right|+1 \\
\leqslant & \frac{1}{2}\left|x_{1}-y_{1}\right|^{2}+\frac{1}{2}\left|x_{2}-y_{2}\right|^{2}+\left|x_{1}-y_{1}\right| \frac{\left|\bar{x}_{1}-y_{1}\right|}{\sqrt{\alpha}}+\left|x_{2}-y_{2}\right| \frac{\left|\bar{x}_{2}-y_{2}\right|}{\sqrt{\alpha}} \\
& \quad+\frac{1}{\alpha}\left|\bar{x}_{2}-y_{2}\right|\left|\bar{x}_{1}-y_{1}\right| \leqslant m_{\alpha}\left(\left|x_{1}-y_{1}\right|^{2}+\left|x_{2}-y_{2}\right|^{2}\right),
\end{aligned}
$$

where $m_{\alpha}=1 / 2+2 / \sqrt{\alpha}+2 / \alpha$. Therefore we compute

$$
\begin{aligned}
\sigma F\left(y_{1} \wedge\right. & \left.y_{2}\right) \sum_{i=1}^{2}\left|x_{i}-y_{i}\right|^{2}+\left.D F\right|_{y_{1} \wedge y_{2}}\left(y_{1} \wedge x_{2}+x_{1} \wedge y_{2}\right)-F\left(y_{1} \wedge y_{2}\right)-F\left(\frac{x_{1} \wedge x_{2}}{\left|x_{1} \wedge x_{2}\right|}\right)\left|x_{1} \wedge x_{2}\right| \\
\geqslant & \sigma \inf _{S^{1}} F \sum_{i=1}^{2}\left|x_{i}-y_{i}\right|^{2}-\sup _{S^{1}}|D F|\left(\left|x_{2}\right|+\left|x_{1}\right|\right)-\sup _{S^{1}} F\left(1+m_{\alpha}\left(\left|x_{1}-y_{1}\right|^{2}+\left|x_{2}-y_{2}\right|^{2}\right)\right) \\
\geqslant & \left(\sigma \inf _{S^{1}} F-m_{\alpha} \sup _{S^{1}} F\right) \sum_{i=1}^{2}\left|x_{i}-y_{i}\right|^{2} \\
& -\sup _{S^{1}}|D F|\left(\left|x_{2}-y_{2}\right| \frac{\left|\bar{x}_{2}-y_{2}\right|}{\sqrt{\alpha}}+\left|x_{1}-y_{1}\right| \frac{\left|\bar{x}_{1}-y_{1}\right|}{\sqrt{\alpha}}+\frac{2}{\alpha}\left|\bar{x}_{2}-y_{2}\right|\left|\bar{x}_{1}-y_{1}\right|\right) \\
& -\sup _{S^{1}} F \frac{1}{\alpha}\left|\bar{x}_{2}-y_{2}\right|\left|\bar{x}_{1}-y_{1}\right| \\
\geqslant & \left(\sigma \inf _{S^{1}} F-m_{\alpha} \sup _{S^{1}} F-\sup _{S^{1}}|D F|\left(\frac{2}{\sqrt{\alpha}}+\frac{4}{\alpha}\right)-\underset{S^{1}}{\alpha} F \frac{2}{\alpha}\right) \sum_{i=1}^{2}\left|x_{i}-y_{i}\right|^{2} \\
\geqslant & \left(\sigma \inf _{S^{1}} F-\max \left\{\sup _{S^{1}} F, \sup _{S^{1}}|D F|\right\}\left(\frac{1}{2}+\frac{4}{\sqrt{\alpha}}+\frac{8}{\alpha}\right)\right) \sum_{i=1}^{2}\left|x_{i}-y_{i}\right|^{2},
\end{aligned}
$$

which is positive for an appropriate choice of $\sigma$.

Case (c): Assume now that $\left|\bar{x}_{2}-y_{2}\right|^{2} \geqslant \alpha$, whereas $\left|\bar{x}_{1}-y_{1}\right|^{2}<\alpha$ for some $4>\alpha>0$ to be chosen later. Obviously $1 / \alpha \geqslant 1 /\left|\bar{x}_{2}-y_{2}\right|^{2}$. In this case

$$
\begin{aligned}
\left|x_{1} \wedge x_{2}\right| \leqslant & \left|x_{1}\right|\left|x_{2}\right| \leqslant\left|x_{1}-y_{1}\right|\left|x_{2}-y_{2}\right|+\left|x_{1}-y_{1}\right|+\left|x_{2}-y_{2}\right|+1 \\
\leqslant & \frac{1}{2}\left|x_{1}-y_{1}\right|^{2}+\frac{1}{2}\left|x_{2}-y_{2}\right|^{2}+\left|x_{1}-y_{1}\right| \frac{\left|\bar{x}_{2}-y_{2}\right|}{\sqrt{\alpha}} \\
& +\left|x_{2}-y_{2}\right| \frac{\left|\bar{x}_{2}-y_{2}\right|}{\sqrt{\alpha}}+\frac{1}{\alpha}\left|\bar{x}_{2}-y_{2}\right|^{2} \\
\leqslant & \left(\frac{1}{2}+\frac{1}{\sqrt{\alpha}}\right)\left|x_{1}-y_{1}\right|^{2}+\left(\frac{1}{2}+\frac{3}{\sqrt{\alpha}}+\frac{4}{\alpha}\right)\left|x_{2}-y_{2}\right|^{2} \\
\leqslant & n_{\alpha} \sum_{i=1}^{2}\left|x_{i}-y_{i}\right|^{2},
\end{aligned}
$$


where $n_{\alpha}=1 / 2+3 / \sqrt{\alpha}+4 / \alpha$. We compute

$$
\begin{aligned}
\sigma F\left(y_{1} \wedge y_{2}\right) \sum_{i=1}^{2}\left|x_{i}-y_{i}\right|^{2}+ & \left.D F\right|_{y_{1} \wedge y_{2}}\left(y_{1} \wedge x_{2}+x_{1} \wedge y_{2}\right)-F\left(y_{1} \wedge y_{2}\right)-F\left(\frac{x_{1} \wedge x_{2}}{\left|x_{1} \wedge x_{2}\right|}\right)\left|x_{1} \wedge x_{2}\right| \\
\geqslant & \sigma \inf _{S^{1}} F \sum_{i=1}^{2}\left|x_{i}-y_{i}\right|^{2}+D F_{y_{1} \wedge y_{2}}\left(y_{1} \wedge\left(x_{2}-y_{2}\right)\right) \\
& +\left|x_{1}\right| D F_{y_{1} \wedge y_{2}}\left(\bar{x}_{1} \wedge y_{2}\right)-F\left(\frac{x_{1} \wedge x_{2}}{\left|x_{1} \wedge x_{2}\right|}\right)\left|x_{1} \wedge x_{2}\right| \\
\geqslant & \left(\sigma \inf _{S^{1}} F-n_{\alpha} \sup _{S^{1}} F\right) \sum_{i=1}^{2}\left|x_{i}-y_{i}\right|^{2}-\sup _{S^{1}}|D F|\left|x_{2}-y_{2}\right| \frac{\left|\bar{x}_{2}-y_{2}\right|}{\sqrt{\alpha}} \\
& -\sup _{S^{1}}|D F|\left(\left|x_{1}-y_{1}\right| \frac{\left|\bar{x}_{2}-y_{2}\right|}{\sqrt{\alpha}}+\frac{\left|\bar{x}_{2}-y_{2}\right|^{2}}{\alpha}\right) \\
\geqslant & \left(\sigma \inf _{S^{1}} F-n_{\alpha} \sup _{S^{1}} F-\sup _{S^{1}}|D F|\left(\frac{3}{\sqrt{\alpha}}+\frac{4}{\alpha}\right)\right) \sum_{i=1}^{2}\left|x_{i}-y_{i}\right|^{2} \\
\geqslant & \left(\sigma \inf _{S^{1}} F-\max \left\{\sup _{S^{1}} F, \sup _{S^{1}}|D F|\right\}\left(\frac{1}{2}+\frac{6}{\sqrt{\alpha}}+\frac{8}{\alpha}\right)\right) \sum_{i=1}^{2}\left|x_{i}-y_{i}\right|^{2},
\end{aligned}
$$

which is positive when $\sigma$ is chosen sufficiently large.

Case $(d)$ : Assume now that $\left|\bar{x}_{1}-y_{1}\right|^{2} \geqslant \alpha$, whereas $\left|\bar{x}_{2}-y_{2}\right|^{2}<\alpha$ for some $4>\alpha>0$. By symmetry this case is handled as Case (c) and the same results are achieved.

Step 4: Putting together the results obtained in Cases (a)-(d), and taking into account that for $0<\alpha<2-\sqrt{2}$ we have $5 /(2-\alpha) \leqslant 6 / \sqrt{\alpha}+8 / \alpha$, we deduce that the proposition is proved provided that we find $\sigma>0$ such that for some fixed $0<\alpha<2-\sqrt{2}$ we have

$$
\sigma \inf _{S^{1}} F \geqslant \max \left\{\sup _{S^{1}} F, \sup _{S^{1}}|D F| \sup _{S^{1}}\left|D^{2} F\right|\right\}\left(\frac{1}{2}+\frac{6}{\sqrt{\alpha}}+\frac{8}{\alpha}\right) .
$$

The constant $\tilde{c}=1 / 2+6 / \sqrt{\alpha}+8 / \alpha$ is minimized for $\alpha \rightarrow 2-\sqrt{2}$. At $\alpha=2-\sqrt{2}$ we have $\tilde{c}=21.99623202$.

\section{Numerical tests}

In the following $\tau$ denotes the time step, $h$ the maximal grid size of the initial discrete surface, and for $p, q \in \mathbb{R}^{3},(p \wedge q)_{1}=\left\langle p \wedge q, e_{1} \wedge e_{2}\right\rangle,(p \wedge q)_{2}=\left\langle p \wedge q, e_{1} \wedge e_{3}\right\rangle,(p \wedge q)_{3}=\left\langle p \wedge q, e_{2} \wedge e_{3}\right\rangle$, where $\left\{e_{1}, e_{2}, e_{3}\right\}$ is the canonical orthonormal basis in $\mathbb{R}^{3}$.

Before we consider the numerical tests, let us briefly remark how to identify the Wulff shape when working in the three-dimensional space.

First of all recall that (in $\mathbb{R}^{3}$ ) Wulff shapes $\mathcal{W}$ are known to be solutions of an isoperimetric problem, i.e. the boundary of $\mathcal{W}$ is the minimizer of 2.1) in the class of surfaces enclosing the same volume (see [7] and references therein). For a flow which, up to tangential components and a 
mobility factor, coincides with the one considered in this paper, it is also known that Wulff shapes shrink self-similarly (see [9, §8.4], [10, pp. 78-79]).

In Section 3 we used the Hodge operator $*$ to define an anisotropy function $\tilde{F}: \mathbb{R}^{3} \rightarrow \mathbb{R}$, $\tilde{F}=F \circ *$, and recover the more familiar formulation of the energy functional $|M|_{F}=\int_{M} \tilde{F}(v) \mathrm{d} A$ (where $v$ is the unit normal to $M$ ). For a convex map $\tilde{F}: \mathbb{R}^{3} \rightarrow[0, \infty)$ such that $\tilde{F}(\lambda y)=|\lambda| \tilde{F}(y)$ (here $y \in \mathbb{R}^{3}$ and $\lambda \in \mathbb{R}$ ), it is well known that the Wulff shape is the convex set with support function $\tilde{F}$, namely

$$
\mathcal{W}_{\tilde{F}}=\left\{x \in \mathbb{R}^{3}: x \cdot y \leqslant \tilde{F}(y) \forall y \in \mathbb{R}^{3}\right\} .
$$

If moreover $\tilde{F}$ is regular, i.e.the unit ball $B_{\tilde{F}}=\left\{x \in \mathbb{R}^{3}: \tilde{F}(x) \leqslant 1\right\}$ has boundary of class $C^{\infty}$ and each principal curvature of $\partial B_{\tilde{F}}$ is strictly positive at each point of $\partial B_{\tilde{F}}$, then the boundary of the Wulff shape is easily determined through

$$
\partial \mathcal{W}_{\tilde{F}}=\nabla \tilde{F}\left(S^{2}\right)
$$

(see [5, §2]).

The above considerations give us a simple means by which we can easily identify the Wulff shape for a given map $F$. For example, for the anisotropy

$$
F(p \wedge q)=\sqrt{\left(a(p \wedge q)_{1}\right)^{2}+\left(b(p \wedge q)_{2}\right)^{2}+\left(c(p \wedge q)_{3}\right)^{2}}
$$

with $a, b, c>0$, we have $(p \wedge q)_{1}=\left\langle p \wedge q, e_{1} \wedge e_{2}\right\rangle=*(p \wedge q) \cdot *\left(e_{1} \wedge e_{2}\right)=*(p \wedge q) \cdot e_{3}$, $(p \wedge q)_{2}=-*(p \wedge q) \cdot e_{2},(p \wedge q)_{3}=*(p \wedge q) \cdot e_{1}$, and, by setting $x=*(p \wedge q) \in \mathbb{R}^{3}$, we obtain

$$
\tilde{F}(x)=\sqrt{c^{2} x_{1}^{2}+b^{2} x_{2}^{2}+a^{2} x_{3}^{2}}
$$

with Wulff shape

$$
\mathcal{W}=\left\{x \in \mathbb{R}^{3}: \sqrt{x_{1}^{2} / c^{2}+x_{2}^{2} / b^{2}+x_{3}^{2} / a^{2}} \leqslant 1\right\} .
$$

In the case $n>3$, borrowing some ideas from [1, \&6.3], one can find a subset of $\Lambda^{n-2} \mathbb{R}^{n}$ that generalizes the definition of Wulff shape as known in the three-dimensional case (see Section 9 . However, it is not clear yet whether this set has some outstanding properties such as those described above for the well studied Wulff shape in $\mathbb{R}^{3}$.

To perform the tests below we used a program that implements Algorithm 6.3 .

Tests 1 and 2 show that a condition on the size of the parameter $\sigma$ is necessary for stability.

Test 1: On the instability of Algorithm 6.1

Starting from a triangulated unit sphere in $\mathbb{R}^{3}$, with time step $\tau=0.1 h^{3}(h=0.286640866)$, we test the energy

$$
E(k)=\int_{M_{h}^{(k)}} F\left(\frac{\partial_{1}^{(k)} \wedge \partial_{2}^{(k)}}{\left|\partial_{1}^{(k)} \wedge \partial_{2}^{(k)}\right|}\right) \mathrm{d} A
$$

over the evolving time for different anisotropies of the form

$$
F(p \wedge q)=\sqrt{\left(a(p \wedge q)_{1}\right)^{2}+\left((p \wedge q)_{2}\right)^{2}+\left((p \wedge q)_{3}\right)^{2}}, \quad a>0 .
$$

The parameter $\sigma$ is chosen to be equal to one, so that we recover Algorithm 6.1 


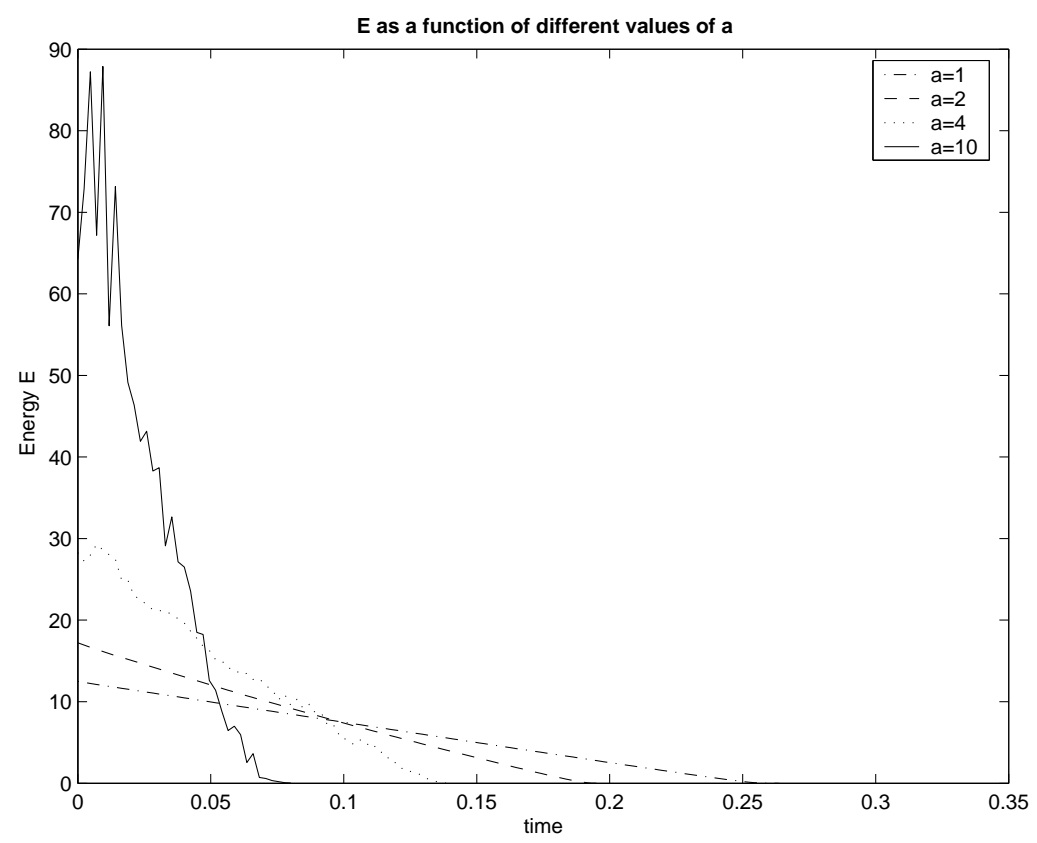

FIG. 1. For Algorithm 6.1 we test the energy over the evolving time and for different anisotropies (Test 1).

The results of the test are shown in Figure 11. Note that for $a=1$ we recover the isotropic case. Although when $a=2$ the energy steadily decreases, graphically a corrugation of the surface is already present. This "corrugation phenomenon" becomes stronger with $a$ increasing, i.e. for "stronger" anisotropies. In Figure 2 we show a picture of the evolved discrete sphere just after a few time steps with the choice of $a=4$.

\section{Test 2: Energy in dependence of the parameter $\sigma$}

For a given map $\Phi: \mathbb{R}^{3} \rightarrow[0, \infty), \Phi(p)=\sqrt{a^{2} p_{1}^{2}+p_{2}^{2}+p_{3}^{2}}$ with $a \geqslant 1$, it is not difficult to show that $\sup _{|p|=1} \Phi(p)=a, \inf _{|p|=1} \Phi(p)=1, \sup _{|p|=1}\left|\Phi^{\prime}(p)\right|=a$ and $\sup _{|p|=1}\left|\Phi^{\prime \prime}(p)\right|=a^{2}$, where $\Phi^{\prime}, \Phi^{\prime \prime}$ denote the gradient and Hessian respectively. In particular, this implies that, for a three-dimensional anisotropy of type (8.4) with $a \geqslant 1$, condition (7.1) reads $\sigma \geqslant 22 a^{2}$. For the case where $a=10$ we look at the evolution of the energy $E(k)$ for different choices of $\sigma$. As in Test 1 we start from a unit sphere in $\mathbb{R}^{3}$ and choose $\tau$ and $h$ to be as in the previous experiment. We test for $\sigma=1,5,15,30,100$. Graphically, for $\sigma=1$ and $\sigma=5$ very strong formation of "wrinkles" can be observed. For $\sigma=15$ the corrugation effect is already extremely weak, and it is no longer present for $\sigma=30$ and $\sigma=100$. We also note that for $\sigma=30$ long triangles are visible in the grid towards the end of the evolution, whereas for $\sigma=100$ this effect is much less visible. The evolution of the energy is shown in Figure 3 .

Although for a choice of large $\sigma$ stability is guaranteed and an improvement in the evolution of the grid is generally observed, we clearly introduce an error (we basically modify the original flow). Looking at the error estimates for the discrete anisotropic mean curvature flow of the graphs 


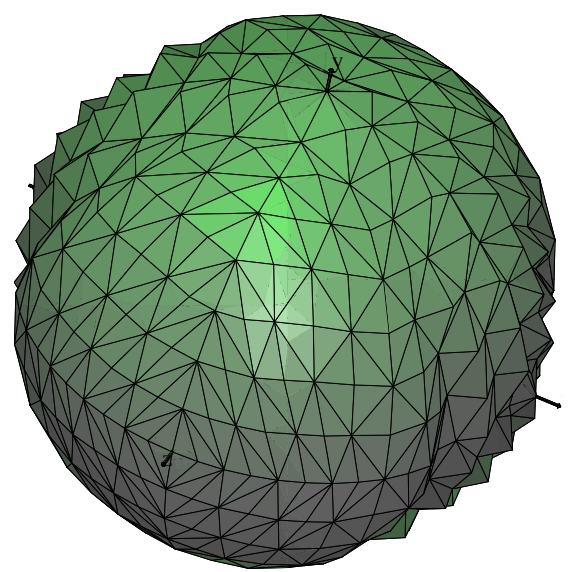

FIG. 2. When stability is not guaranteed, a "corrugation" phenomenon can be observed just after a few time steps (Test 1).

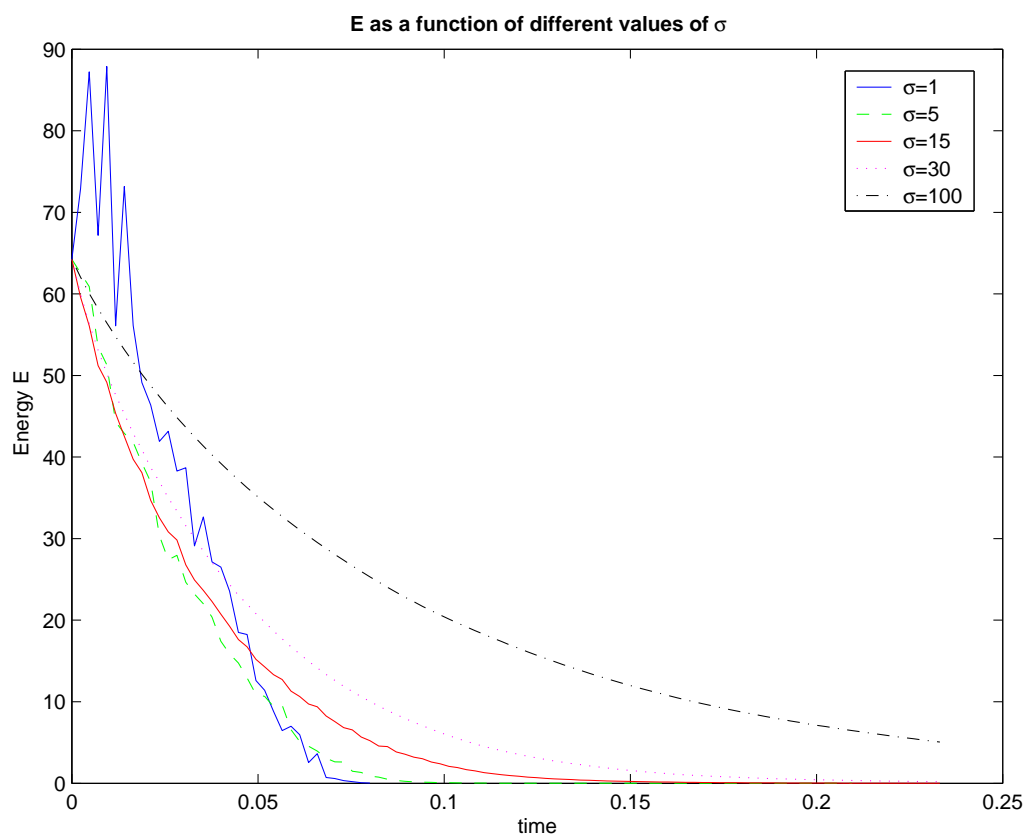

FIG. 3. For Algorithm 6.3 we test the energy over the evolving time and for different choices of $\sigma$ (Test 2).

given in [8], we may think that this error can be compensated for by choosing a very small grid size and time step. In fact by fixing the anisotropy function, $h$, and $\sigma$, one can already observe a faster decrease in the energy $E$ by taking smaller and smaller time steps. Small time steps, however, have the nasty effect of introducing rounding errors, so it is advisable to try to keep $\sigma$ "as small as possible".

The fact that in the isotropy case the choice of $\sigma=1$ (see Section 6.1), and not of $\sigma=22$ as in Proposition 7.1, is actually sufficient to guarantee stability, the results for the graph case discussed 
in [8], and several numerical experiments seem to suggest that the multiplying constant 22 in (7.1) is in practice too large. We conjecture that a different stability proof might lead to an improvement of this constant.

In the next experiments the parameter $\sigma$ is mostly chosen with the previous remark in mind: roughly speaking, we replace the constant 22 in $(7.1)$ by something close to one (similarly to the case of graphs in codimension one discussed in [8] and to the case of curves moving by anisotropic mean curvature in $\mathbb{R}^{n}$ treated in [15]). The results are in general satisfactory.

\section{Test 3: Testing against a known solution}

Consider the anisotropy $F(p \wedge q)=|p \wedge q|-0.25(p \wedge q)_{1}$. Here the Wulff shape is a unit ball centered at $C=(0,0,-0.25)$. An exact solution for the flow

$$
\left\{\begin{array}{l}
\mathbf{x}_{t}=H_{F}, \\
\mathbf{x}(0)\left(S^{2}\right)=\operatorname{Id}_{\mathbb{R}^{3}}\left(S^{2}\right),
\end{array}\right.
$$

under the given anisotropy is given by the shrinking unit sphere $x(t, y)=\sqrt{1-4 t} y$, where $y \in S^{2}$. This implies that if we triangulate a unit sphere, then the nodes should move radially towards the origin, following the law $P(t, i)=\sqrt{1-4 t} P(i)$, where $P(i):=\left(p_{1}(i), p_{2}(i), p_{3}(i)\right) \in S^{2}$ is the coordinate vector of node $i \in\{1, \ldots, J\}$. In the following the $L^{\infty}\left(L^{\infty}\right)$-error is

$$
\operatorname{err}_{h}=\max _{t \in[0,0.12)} \max _{i \in J}\left\|P(t, i)-x_{h}(t, i)\right\|_{\mathbb{R}^{3}}
$$

where $x_{h}(t, i)$ denotes the coordinate of node $i$ at time $t$. A quick calculation shows that $\left.\sup F\right|_{S^{1}}=$ $5 / 4,\left.\inf F\right|_{S^{1}}=3 / 4, \sup _{S^{1}}|D F|=5 / 4$ and $\sup _{S^{1}}\left|D^{2} F\right|=1$, so that we choose $\sigma=2$ (recall the above discussion). In the following tables, "final $T$ " is the actual time over which the error is calculated, and eoc is the error of convergence which is calculated by

$$
\mathrm{eoc}=\ln \left(\operatorname{err}_{h_{i}} / \operatorname{err}_{h_{i+1}}\right) / \ln \left(h_{i} / h_{i+1}\right)
$$

for two successive grid sizes $h_{i}$ and $h_{i+1}$ of the initial surface. In Table 1 we show the error calculated for a time step equal to $\tau=0.01 \mathrm{~h}$ : here a linear order of convergence is clearly recognizable. For the choice of $\tau=0.1 h^{2}$, the results shown in Table 2 suggest a better order of convergence. Making the time even smaller leads to results that cannot be clearly interpreted, most probably due to rounding errors entering the computation.

TABLE 1

Anisotropic flow, error for $\sigma=2, \tau=0.01$ (Test 3)

\begin{tabular}{|l|l|l|l|}
\hline$h$ & $L^{\infty}\left(L^{\infty}\right)$ & eoc & final $T$ \\
\hline 0.557452781 & 0.0240660391 & - & 0.117065081 \\
\hline 0.286640866 & 0.00797789406 & 1.6599729554972 & 0.117522752 \\
\hline 0.144333189 & 0.00404673364 & 0.989300150038 & 0.119796544 \\
\hline 0.0722939259 & 0.00196217256 & 1.04696868493074 & 0.119284975 \\
\hline 0.0361629024 & 0.00096201316 & 1.02897785263166 & 0.119699204 \\
\hline
\end{tabular}


TABLE 2

Anisotropic flow, error for $\sigma=2, \tau=0.1 h^{2}$ (Test 3)

\begin{tabular}{|l|l|l|l|}
\hline$h$ & $L^{\infty}\left(L^{\infty}\right)$ & eoc & final $T$ \\
\hline 0.557452781 & 0.0411465787 & - & 0.0932260823 \\
\hline 0.286640866 & 0.0188901754 & 1.17041494029746 & 0.115028182 \\
\hline 0.144333189 & 0.00553169924 & 1.79002594291820 & 0.118742789 \\
\hline 0.0722939259 & 0.00147294533 & 1.91388634787591 & 0.11968483 \\
\hline
\end{tabular}

Test 4: Increasing $\sigma$ in the isotropic setting

The exact solution considered in Test 3 is also a solution to the isotropic mean curvature flow and stability is here demonstrated already for $\sigma=1$. For $h=0.144333189$, linear time step $\tau=0.01 h$, and final time $T=0.119796544$, we look at the error $L^{\infty}\left(L^{\infty}\right)$ as $\sigma$ increases. The results are shown in Table 3 The choice of time step $\tau=0.1 h^{2}$ gives comparable results.

TABLE 3

Isotropic flow, $h=0.144333189, \tau=0.01 h$, final $T=0.119796544$ (Test 4)

\begin{tabular}{|l|l|l|l|}
\hline$\sigma$ & $L^{\infty}\left(L^{\infty}\right)$ & $\sigma$ & $L^{\infty}\left(L^{\infty}\right)$ \\
\hline 1 & 0.00320766272 & 32 & 0.0357178408 \\
\hline 2 & 0.00366413277 & 64 & 0.0609411155 \\
\hline 4 & 0.00617566452 & 128 & 0.0966144499 \\
\hline 8 & 0.0110011148 & 256 & 0.139571567 \\
\hline 16 & 0.0199786682 & 512 & 0.182691568 \\
\hline
\end{tabular}

\section{Graphical examples}

We conclude this section with some graphical examples. First we show the evolution of a threedimensional dumbbell under the anisotropy (8.1) with $\sigma=16$. For $c=4, a=b=1$, four shots of the evolution are shown in Figure 4 For $b=4, a=c=1$ the evolution is shown in Figure 5 (see also Figure 6 .

Using again the anisotropy 8.1 with $a=2, b=c=1$, and choosing $\sigma=4$, we show in Figure 7 the evolution of a torus.

Next we take an ellipsoid implicitly defined by $x^{2}+y^{2}+z^{2} / 4=1$ and let it move under the $l_{4}$-anisotropy

$$
F(p \wedge q)=\left(\left|(p \wedge q)_{1}\right|^{4}+\left|(p \wedge q)_{2}\right|^{4}+\left|(p \wedge q)_{3}\right|^{4}\right)^{1 / 4} .
$$

Here $\sigma=9$, and the evolution is shown in Figure 8

Although crystalline anisotropies are not covered by the theory, we experiment with the $l_{1}$-norm

$$
F(p \wedge q)=\left|(p \wedge q)_{1}\right|+\left|(p \wedge q)_{2}\right|+\left|(p \wedge q)_{3}\right| .
$$

In Figure 9 we show the evolution of a unit sphere; $\sigma=20$ is chosen experimentally, so that no corrugations of the surface appear. In Figure 10 the last shot is rescaled in order to show the triangulation. 

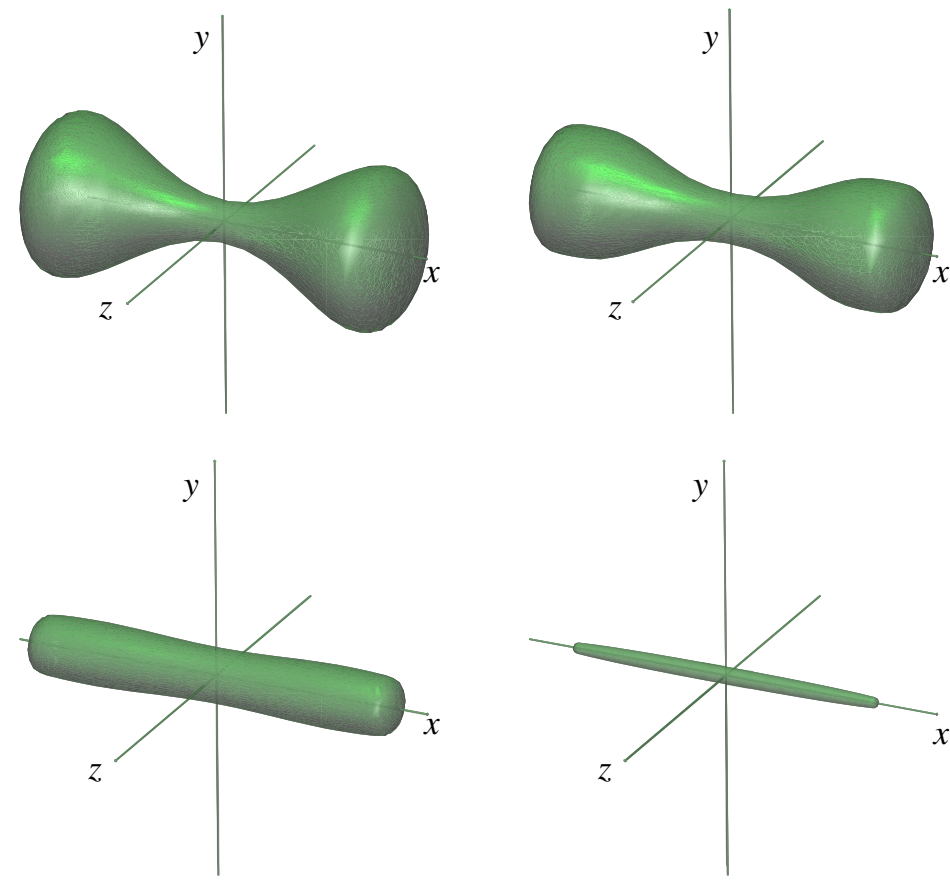

FIG. 4. The Wulff shape is $\mathcal{W}=\left\{x \in \mathbb{R}^{3}: \sqrt{x^{2} / 16+y^{2}+z^{2}} \leqslant 1\right\}$.
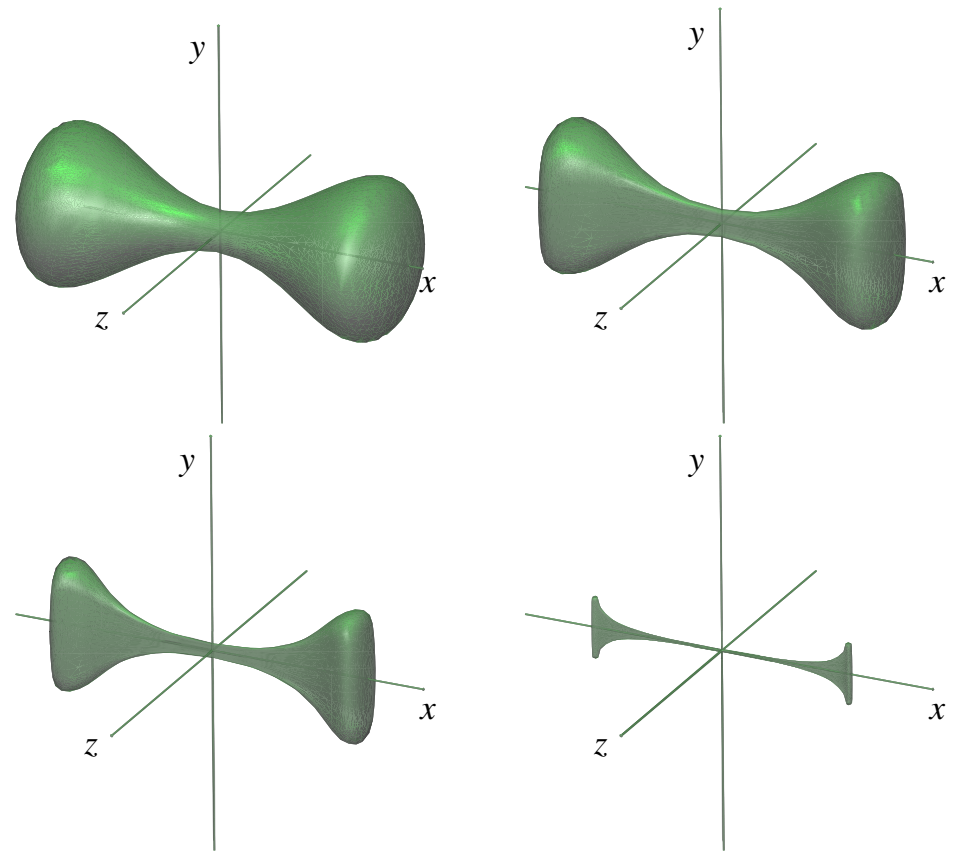

FIG. 5. The Wulff shape is $\mathcal{W}=\left\{x \in \mathbb{R}^{3}: \sqrt{x^{2}+y^{2} / 16+z^{2}} \leqslant 1\right\}$. 


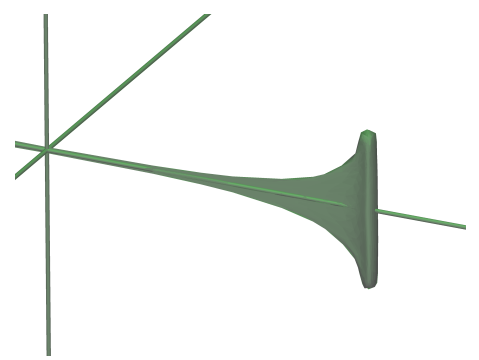

FIG. 6. A detail of the last shot of Figure 5
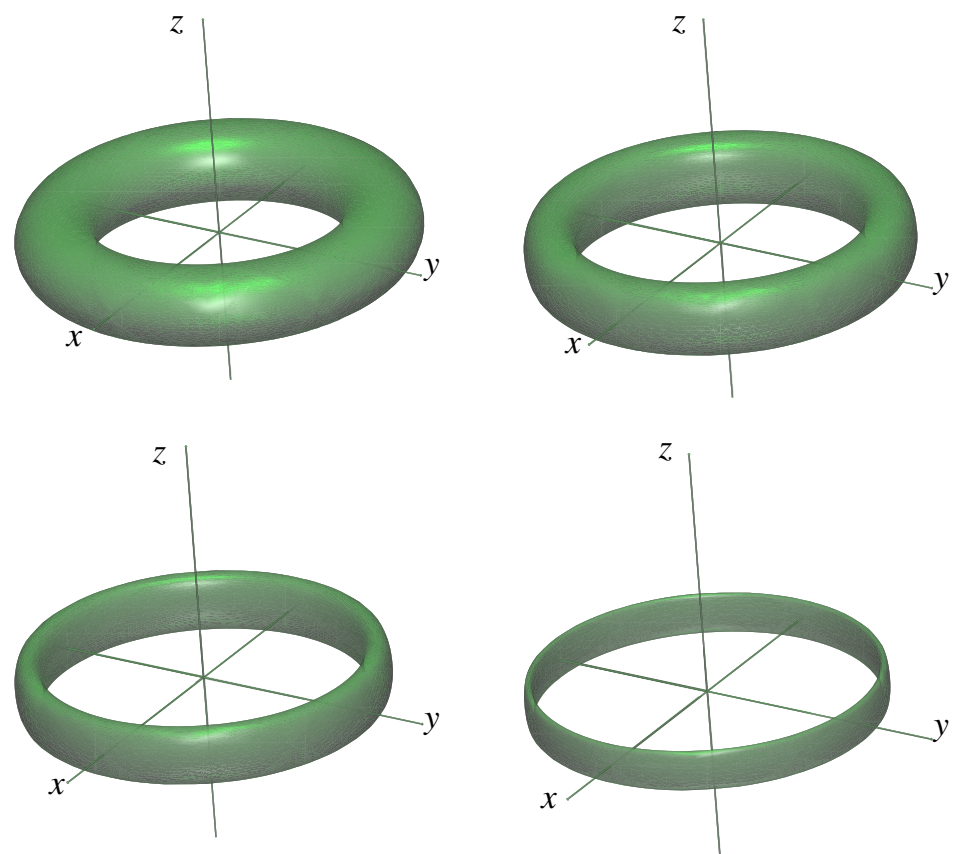

FIG. 7. The Wulff shape is $\mathcal{W}=\left\{x \in \mathbb{R}^{3}: \sqrt{x^{2}+y^{2}+z^{2} / 4} \leqslant 1\right\}$.

In Figure 11 we show the evolution of a dumbbell for the same choice of $\sigma$ and anisotropy function.

\section{Some geometrical remarks}

As mentioned in the introduction, the anisotropic area functional 2.1) can be interpreted as the choice of an area functional for $\mathbb{R}^{n}$ when viewed as a Minkowski space. In this context $F$ is a map that helps describe the ratio between the two-dimensional Minkowski and Lebesgue measures (see [20, Chapter 5] and [1]).

One of the peculiarities of Minkowski geometry is the continuous interplay between the Minkowski space (in our case $\mathbb{R}^{n}$ with an additional norm $\|\cdot\|$ ) and its dual. In the study of the anisotropic curve shortening flow this fact becomes tangible when looking carefully at the equation 

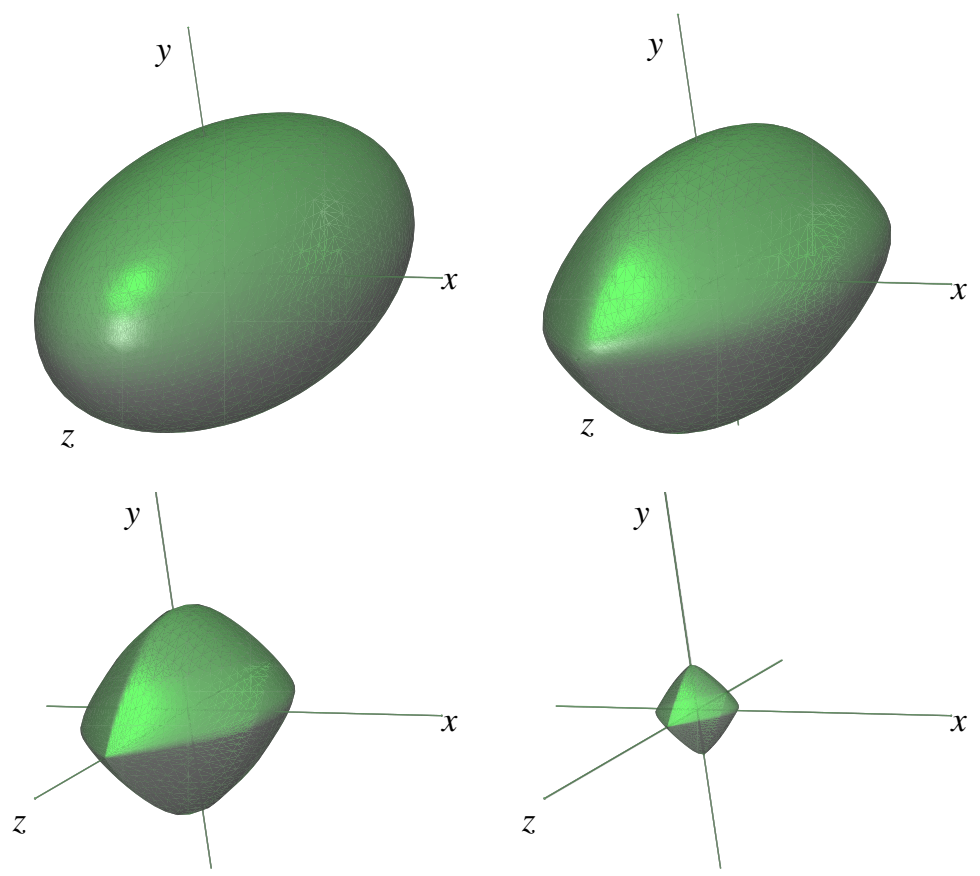

FIG. 8. The Wulff shape is $\left\{x \in \mathbb{R}^{3}:\|x\|_{4 / 3} \leqslant 1\right\}$.
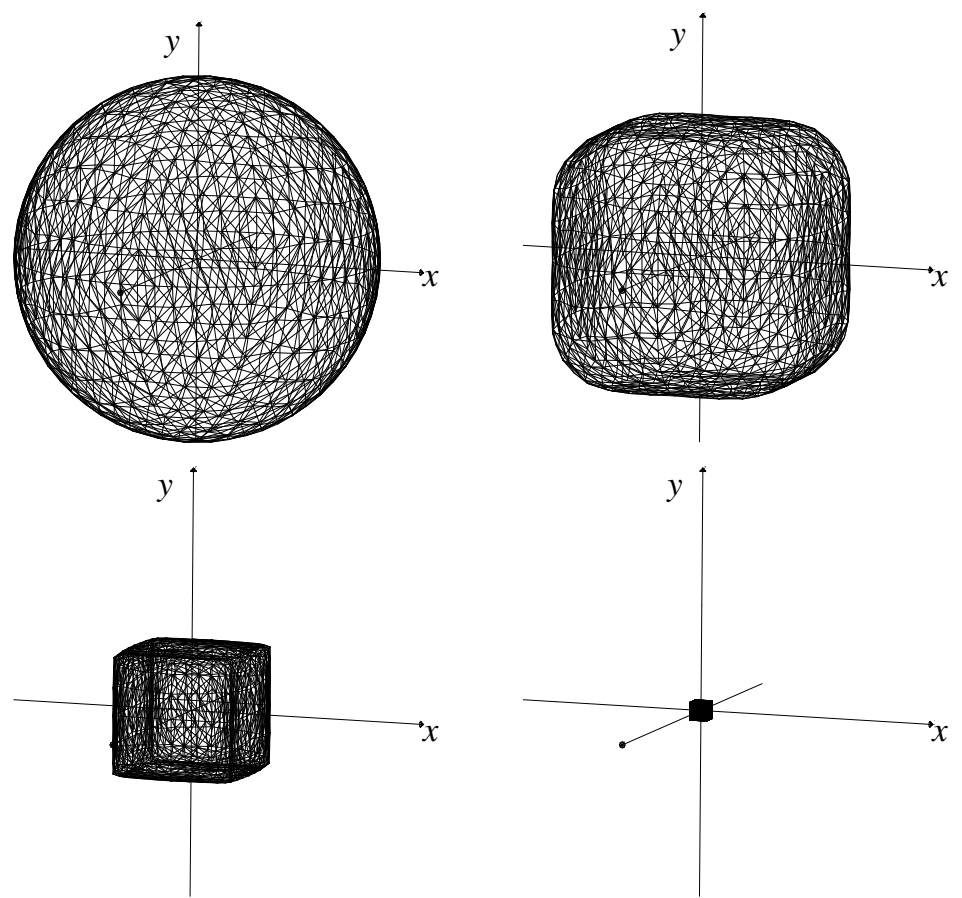

FIG. 9. The Wulff shape is a unit cube. 


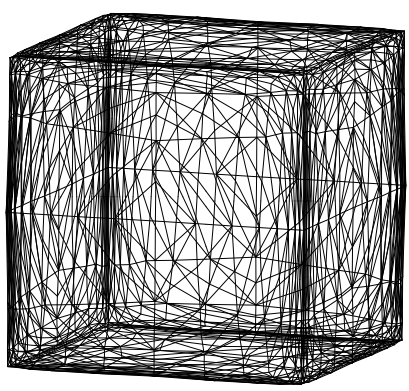

FIG. 10. The last shot of Figure 9 after rescaling.
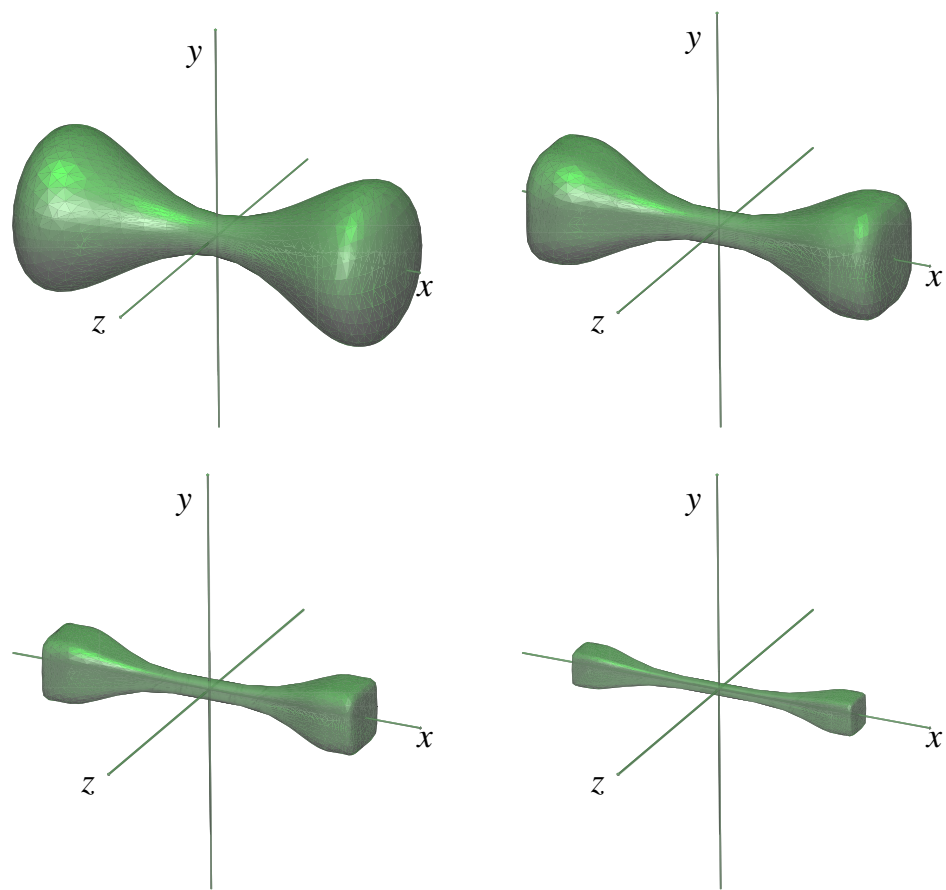

FIG. 11. The Wulff shape is a unit cube.

defining the flow (see [15, §6]). For the case of two-dimensional surfaces things are not quite so straightforward but we can nonetheless make some geometrical remarks that might help the reader recognize such ongoing interplay between dual structures.

Most of the following ideas are borrowed from [1, §6.3]. In $\mathbb{R}^{n}$ (with the canonical oriented basis $\left\{e_{1}, \ldots, e_{n}\right\}$ and inner product) let $\Omega \in\left(\Lambda^{n} \mathbb{R}^{n}\right)^{*}$ be a volume form with $\Omega\left(e_{1} \wedge \cdots \wedge e_{n}\right)$ $=1$. Consider the linear isomorphism

$$
i_{\Omega}: \Lambda^{2} \mathbb{R}^{n} \rightarrow\left(\Lambda^{n-2} \mathbb{R}^{n}\right)^{*}
$$


defined by $i_{\Omega}\left(v_{1} \wedge v_{2}\right)\left(y_{1} \wedge \cdots \wedge y_{n-2}\right)=\Omega\left(v_{1} \wedge v_{2} \wedge y_{1} \wedge \cdots \wedge y_{n-2}\right)$. Using the definition of the Hodge operator

$$
y_{1} \wedge \cdots \wedge y_{n-2} \wedge v_{1} \wedge v_{2}=\left\langle *\left(y_{1} \wedge \cdots \wedge y_{n-2}\right), v_{1} \wedge v_{2}\right\rangle_{\Lambda^{2} \mathbb{R}^{n}} e_{1} \wedge \cdots \wedge e_{n}
$$

(see for example [12, §2.7]), we infer

$$
\begin{aligned}
i_{\Omega}\left(v_{1} \wedge v_{2}\right)\left(y_{1}\right. & \left.\wedge \cdots \wedge y_{n-2}\right)=\Omega\left(v_{1} \wedge v_{2} \wedge y_{1} \wedge \cdots \wedge y_{n-2}\right) \\
& =\Omega\left(y_{1} \wedge \cdots \wedge y_{n-2} \wedge v_{1} \wedge v_{2}\right)=\left\langle *\left(y_{1} \wedge \cdots \wedge y_{n-2}\right), v_{1} \wedge v_{2}\right\rangle_{\Lambda^{2} \mathbb{R}^{n}} \\
& =\left\langle *\left(v_{1} \wedge v_{2}\right), y_{1} \wedge \cdots \wedge y_{n-2}\right\rangle_{\Lambda^{n-2} \mathbb{R}^{n}}
\end{aligned}
$$

For a given norm $F: \Lambda^{2} \mathbb{R}^{n} \rightarrow[0, \infty)$, let

$$
\mathcal{B}:=\left\{v_{1} \wedge v_{2} \in \Lambda^{2} \mathbb{R}^{n}: F\left(v_{1} \wedge v_{2}\right) \leqslant 1\right\}
$$

be the unit ball for $F$ and define $I$ to be the polar of its image under $i_{\Omega}$, i.e. $I:=\left(i_{\Omega} \mathcal{B}\right)^{*}$. We find that $I$ is a symmetric convex set described by

$I=\left\{a_{1} \wedge \cdots \wedge a_{n-2} \in \Lambda^{n-2} \mathbb{R}^{n}: \Omega\left(a_{1} \wedge \cdots \wedge a_{n-2} \wedge v_{1} \wedge v_{2}\right) \leqslant F\left(v_{1} \wedge v_{2}\right), \forall v_{1} \wedge v_{2} \in \Lambda^{2} \mathbb{R}^{n}\right\}$.

Observe that for $n=3, I$ is the well known Wulff shape (to relate the above equation to the more familiar description given for example in [7, §3.2], consider as usual $\tilde{F}=F \circ *: \mathbb{R}^{3} \rightarrow \mathbb{R}$ and (9.2).

Given $v_{1} \wedge v_{2} \in \Lambda^{2} \mathbb{R}^{n} \backslash\{0\}$, we say that $z_{1} \wedge \cdots \wedge z_{n-2} \in \Lambda^{n-2} \mathbb{R}^{n}$ is normal to $v_{1} \wedge v_{2}$ with respect to $I$ if $z_{1} \wedge \cdots \wedge z_{n-2} \in \partial I, \Omega\left(v_{1} \wedge v_{2} \wedge z_{1} \wedge \cdots \wedge z_{n-2}\right)>0$, and the hyperplane passing through $z_{1} \wedge \cdots \wedge z_{n-2} \in \partial I$ and perpendicular to $*\left(v_{1} \wedge v_{2}\right) \in \Lambda^{n-2} \mathbb{R}^{n}$ supports $I$. If $I$ is smooth and strictly convex, the normal is unique. Notice that $z_{1} \wedge \cdots \wedge z_{n-2}$ is constructed in such a way that

$$
\begin{aligned}
& \Omega\left(v_{1} \wedge v_{2} \wedge z_{1} \wedge \cdots \wedge z_{n-2}\right) \\
& \quad=\sup \left\{\left|\Omega\left(v_{1} \wedge v_{2} \wedge a_{1} \wedge \cdots \wedge a_{n-2}\right)\right|: a_{1} \wedge \cdots \wedge a_{n-2} \in I\right\} \\
& \left(\begin{array}{l}
=\sup \left\{\left\langle *\left(v_{1} \wedge v_{2}\right), a_{1} \wedge \cdots \wedge a_{n-2}\right\rangle: a_{1} \wedge \cdots \wedge a_{n-2} \in I\right\} \\
=\left|v_{1} \wedge v_{2}\right| \sup \left\{\left\langle *\left(\frac{v_{1} \wedge v_{2}}{\left|v_{1} \wedge v_{2}\right|}\right), a_{1} \wedge \cdots \wedge a_{n-2}\right\rangle: a_{1} \wedge \cdots \wedge a_{n-2} \in I\right\} \\
\text { and } \left.*\left(\frac{v_{1} \wedge v_{2}}{\left|v_{1} \wedge v_{2}\right|}\right) \text { lies on the unit sphere in } \Lambda^{n-2} \mathbb{R}^{n}\right) .
\end{array}\right.
\end{aligned}
$$

Finally, denote by $\|\cdot\|_{*}$ the norm in $\left(\Lambda^{n-2} \mathbb{R}^{n}\right)^{*}$ whose unit ball is $I^{*}=i_{\Omega}(\mathcal{B})$. Since $\| i_{\Omega}\left(v_{1} \wedge\right.$ $\left.v_{2}\right) \|_{*}=F\left(v_{1} \wedge v_{2}\right)$, we infer that

$$
\begin{aligned}
F\left(v_{1} \wedge v_{2}\right) & =\left\|i_{\Omega}\left(v_{1} \wedge v_{2}\right)\right\|_{*} \\
& =\sup \left\{\left|f\left(i_{\Omega}\left(v_{1} \wedge v_{2}\right)\right)\right|: f \in\left(i_{\Omega} \mathcal{B}\right)^{*}\right\} \quad \text { (by [20, Corollary 1.3.7]) } \\
& =\sup \left\{\left|\Omega\left(v_{1} \wedge v_{2} \wedge a_{1} \wedge \cdots \wedge a_{n-2}\right)\right|: a_{1} \wedge \cdots \wedge a_{n-2} \in I\right\} \\
& =\Omega\left(v_{1} \wedge v_{2} \wedge z_{1} \wedge \cdots \wedge z_{n-2}\right)
\end{aligned}
$$

for $z_{1} \wedge \cdots \wedge z_{n-2}$ normal to $v_{1} \wedge v_{2}$ with respect to $I$. 
Thus we conclude that if $v_{1}, v_{2}$ are linearly independent vectors and $z_{1} \wedge \cdots \wedge z_{n-2}$ is normal to $v_{1} \wedge v_{2}$ with respect to $I$ then the volume of the parallelotope $\left[v_{1}, v_{2}, z_{1}, \ldots, z_{n-2}\right]$ spanned by the vectors $v_{1}, v_{2}, z_{1}, \ldots, z_{n-2}$ is equal to the two-dimensional (anisotropic) area of the parallelogram $\left[v_{1}, v_{2}\right]$, i.e.

$$
\Omega\left(v_{1} \wedge v_{2} \wedge z_{1} \wedge \cdots \wedge z_{n-2}\right)=F\left(v_{1} \wedge v_{2}\right) .
$$

\section{Acknowledgments}

I would like to thank Professor Gerhard Dziuk and Professor Klaus Deckelnick for helpful discussions, and Professor Dziuk for making available to me some of his subroutines. The work was partially supported by the Deutsche Forschungsgemeinschaft via DFG-Forschergruppe "Nonlinear Partial Differential Equations: Theoretical and Numerical Analysis". The graphics were created with the packages GRAPE and Matlab.

\section{REFERENCES}

1. Álvarez Paiva, J. C., \& Thompson, A. C. Volumes on normed and Finsler spaces. A Sampler of Riemann-Finsler Geometry, Math. Sci. Res. Inst. Publ. 50, Cambridge Univ. Press, Cambridge (2004), 1-48. Zbl 1078.53072 MR 2132656

2. Ambrosio, L., \& Soner, H. M. Level set approach to mean curvature flow in arbitrary codimension. J. Differential Geom. 43 (1996), 693-737. Zbl 0868.35046 MR 1412682

3. Andrews, B. Volume-preserving anisotropic mean curvature flow. Indiana Univ. Math. J. 50 (2001), 783-827. Zbl 1047.53037 MR 1871390

4. B ̈̈NSCH, E. Finite element discretization of the Navier-Stokes equations with a free capillary surface. Numer. Math. 88 (2001), 203-235. Zbl 0985.35060 MR 1826211

5. Bellettini, G. Anisotropic and crystalline mean curvature flow. A Sampler of Riemann-Finsler Geometry, Math. Sci. Res. Inst. Publ. 50, Cambridge Univ. Press, Cambridge (2004), 49-82. Zbl 1086.53083 MR 2132657

6. Clarenz, U. Enclosure theorems for extremals of elliptic parametric functionals. Calc. Var. Partial Differential Equations 15 (2002), 313-324. Zbl 1018.53006 MR 1938817

7. Clarenz, U., Dziuk, G., \& RUMPF, M. On generalized mean curvature flow in surface processing. Geometric Analysis and Nonlinear Partial Differential Equations. Springer, Berlin (2003), 217-248. Zbl 1035.53091 MR 2008341

8. DeCKelnick, K., \& DZIUK, G. A fully discrete numerical scheme for weighted mean curvature flow. Numer. Math. 91 (2002), 423-452. Zbl 0999.65103 MR 1907866

9. Deckelnick, K., Dziuk, G., \& Elliott, C. M. Computation of geometric partial differential equations and mean curvature flow. Acta Numer. 14 (2005), 139-232. Zbl 1113.65097 MR 2168343

10. Diewald, U. Anisotrope Krümmungsflüsse parametrischer Fläche sowie deren Anwendung in der Flächenverarbeitung. Dissertation (2005).

11. Do CARmo, M. P. Riemannian Geometry. Birkhäuser Boston, Boston, MA (1992). Zbl 0752.53001 MR 1138207

12. Flanders, H. Differential Forms with Applications to the Physical Sciences. Academic Press, New York (1963). Zbl 0733.53002 MR 0162198

13. Gallot, S., Hulin, D., \& Lafontaine, J. Riemannian Geometry, 2nd ed., Springer, Berlin (1990). Zbl 0716.53001 MR 1083149

14. Hildebrandt, S., \& Von Der Mosel, H. Conformal representation of surfaces, and Plateau's problem for Cartan functionals. Riv. Mat. Univ. Parma (7) 4* (2005), 1-43. Zbl 1107.49015 MR 2197479 
15. PozZI, P. Anisotropic curve shortening flow in higher codimension. Math. Methods Appl. Sci. 30 (2007), 1243-1281. Zbl 1122.65081 MR 2334978

16. SERnesi, E. Geometria 2. Bollati Boringhieri, Torino (1994).

17. Simon, L. Lectures on Geometric Measure Theory. Proc. Centre Math. Anal. Austral. National Univ. 3 , Austral. National Univ., Canberra (1983). Zbl 0546.49019 MR 0756417

18. SLEPČEV, D. On level-set approach to motion of manifolds of arbitrary codimension. Interfaces Free Bound. 5 (2003), 417-458. Zbl 1040.35126 MR 2031465

19. Taylor, M. E. Partial Differential Equations I, Basic Theory. Appl. Math. Sci. 115, Springer (1997). Zbl 0869.35002 MR 1395147

20. Thompson, A. C. Minkowski Geometry. Encyclopedia Math. Appl. 63, Cambridge Univ. Press, Cambridge (1996). Zbl 0868.52001 MR 1406315

21. Vinberg, E. B. A Course in Algebra. Grad. Stud. Math. 56, Amer. Math. Soc., Providence, RI (2003). Zbl 1016.00003 MR 1974508

22. WAng, M.-T. Mean curvature flow in higher codimension. Second Int. Congress of Chinese Mathematicians (2002), to appear.

23. Willmore, T. J. Riemannian Geometry. Oxford Sci. Publ., Clarendon Press, New York (1993). Zbl 0797.53002 MR 1261641 